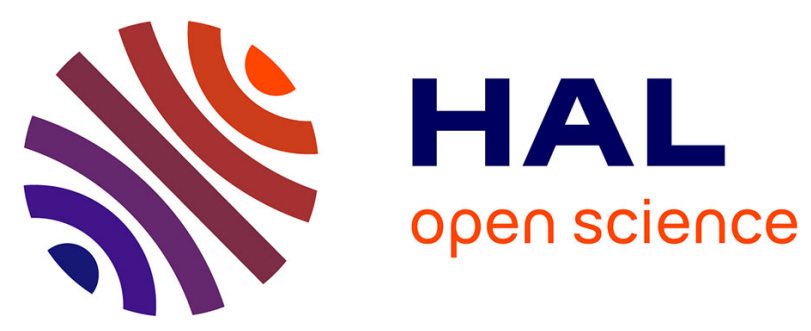

\title{
Fast high-resolution brain metabolite mapping on a clinical 3T MRI by accelerated 1 H-FID-MRSI and low-rank constrained reconstruction
}

Antoine Klauser, Sebastien Courvoisier, Jeffrey Kasten, Michel Kocher, Matthieu Guerquin-Kern, Dimitri van de Ville, François Lazeyras

\section{To cite this version:}

Antoine Klauser, Sebastien Courvoisier, Jeffrey Kasten, Michel Kocher, Matthieu Guerquin-Kern, et al.. Fast high-resolution brain metabolite mapping on a clinical 3T MRI by accelerated 1 H-FID-MRSI and low-rank constrained reconstruction. Magnetic Resonance in Medicine, 2018. hal-01960235

\section{HAL Id: hal-01960235 https://hal.science/hal-01960235}

Submitted on 20 Dec 2018

HAL is a multi-disciplinary open access archive for the deposit and dissemination of scientific research documents, whether they are published or not. The documents may come from teaching and research institutions in France or abroad, or from public or private research centers.
L'archive ouverte pluridisciplinaire HAL, est destinée au dépôt et à la diffusion de documents scientifiques de niveau recherche, publiés ou non, émanant des établissements d'enseignement et de recherche français ou étrangers, des laboratoires publics ou privés. 


\title{
Fast high-resolution brain metabolite mapping on a clinical 3T MRI by accelerated ${ }^{1}$ H-FID-MRSI and low-rank constrained reconstruction
}

\author{
Antoine Klauser ${ }^{1}$, Sebastien Courvoisier ${ }^{1}$, Jeffrey Kasten ${ }^{1}$,Michel Kocher ${ }^{1}$, \\ Matthieu Guerquin-Kern ${ }^{3}$, Dimitri Van De Ville ${ }^{1,2}$, Franccois Lazeyras ${ }^{1}$ \\ ${ }^{1}$ Department of Radiology and Medical Informatics, University of Geneva, Switzerland \\ ${ }^{2}$ Institute of Bioengineering, Ecole Polytechnique Federale de Lausanne, Switzerland and \\ ${ }^{3}$ ETIS UMR 8051, ENSEA, Université de Cergy-Pontoise, CNRS, France
}

(Dated: December 20, 2018)

\begin{abstract}
Purpose Epitomizing the advantages of ultra short echo time and no chemical shift displacement error, high-resolution free induction decay magnetic resonance spectroscopic imaging (FIDMRSI) sequences have proven to be highly effective in providing unbiased characterizations of metabolite distributions. However, its merits are often overshadowed in high-resolution settings by reduced signal-to-noise ratios resulting from the smaller voxel volumes procured by extensive phase encoding and the related acquisition times.

Methods To address these limitations, we here propose an acquisition and reconstruction scheme that offers both implicit dataset denoising and acquisition acceleration. Specifically, a slice selective high-resolution FID-MRSI sequence was implemented. Spectroscopic datasets were processed to remove fat contamination, and then reconstructed using a total generalized variation (TGV) regularized low-rank model. We further measured reconstruction performance for random undersampled data to assess feasibility of a compressed-sensing SENSE acceleration scheme. Performance of the lipid suppression was assessed using an ad hoc phantom, while that of the low-rank TGV reconstruction model was benchmarked using simulated MRSI data. To assess real-world performance, 2D FID-MRSI acquisitions of the brain in healthy volunteers were reconstructed using the proposed framework.

Results Results from the phantom and simulated data demonstrate that skull lipid contamination is effectively removed and that data reconstruction quality is improved with the low-rank TGV model. Also, we demonstrated that the presented acquisition and reconstruction methods are compatible with a compressed-sensing SENSE acceleration scheme.

Conclusion An original reconstruction pipeline for $2 \mathrm{D}{ }^{1} \mathrm{H}-\mathrm{FID}-\mathrm{MRSI}$ datasets was presented that places high-resolution metabolite mapping on 3T MR scanners within clinically feasible limits.
\end{abstract}

\section{INTRODUCTION}

Proton magnetic resonance spectroscopic imaging ${ }^{1} H$ MRSI is an advanced MR technique capable of measuring spectral content corresponding to distinct metabolites [1]. Through spectral dataset encoded in space with phase encoding in 2 or 3 dimensions, the technique provides rich information over the composition of the tissue under observation and their metabolite distribution. However, traditional acquisition and reconstruction techniques have typically been circumscribed by the limited available signal resulting from low metabolite concentrations, ultimately culminating in lengthy acquisition durations and severely constrained image resolutions. As an added consequence, the signal content associated with the resulting coarse voxels often reflects contributions from several tissue types, thereby limiting accurate quantification. The ability to increase image resolution without adversely affecting the signal quality would therefore represent a significant step forward.

Recent ${ }^{1} H$-FID-MRSI sequences has proven to be particularly effective for measuring high-resolution $2 \mathrm{D}$ metabolic distributions across the whole brain at ultrahigh field [2-6]. Combining the advantages of ultra short echo time, i.e. preserving the signal of strongly J-coupled metabolites, and the absence of chemical shift displacement errors, the technique provides an unbiased charac- terization of the metabolite distribution. Moreover, the simple sequence design permits to reduce considerably the acquisition time by decreasing repetition time while using optimal Ernst's flip angle. This novel approach in metabolic imaging enables previously unachievable spatial resolutions, revealing metabolic differences between brain tissues [7, 8] at ultra-high field. However,at field strengths typically utilized in clinical settings (3T), highresolution metabolic imaging suffers from weak signalto-noise ratios (SNR) due to the small voxel volume $(\sim 0.1 \mathrm{ml})$, leading to noisy metabolite distribution images. Nevertheless, reconstruction methods based on low-rank assumptions are able to efficiently denoise MRSI dataset using partial separability $[9,10]$. Also, in combination with accelerated acquisitions schemes, methods exploiting the spatio-temporal correlations permit reconstruction of high-resolution metabolite images [11-13] in addition to nuisance signal removal $[14,15]$ and fast phosphorus MRSI acquisition [16]. These approaches efficiently denoise MRSI dataset while preserving metabolite distribution features [17]. The aim of the research presented in this study is to explore possibilities of highresolution MRSI accelerated with a compressed-sensing SENSE scheme while combining and adapting existing methods for acquisition, lipid suppression and reconstruction. To this end, a ${ }^{1} H$-FID-MRSI acquisition scheme [2] was combined with the lipid suppression by 
orthogonality method [18] and low-rank total generalized variation (TGV) reconstruction $[19,20]$. The reconstruction of accelerated parallel MRSI was designed building on previous framework established by Kasten et $a l .[19]$. This model has been extended to include separately signal from each coil element with corresponding sensitivity profile and to perform reconstruction on undersampled k-space data. We first demonstrate effective lipid suppression on an in-house fat phantom using an optimized with the optimized metabolite -lipid orthogonality method. Performance of the low-rank TGV model is then assessed using simulated data from an analytical phantom. Metabolite distributions of healthy volunteers resulting from the whole reconstruction pipeline are then presented. In addition, SENSE acceleration along with variable density random undersampling of the k-space deemed compressed-sensing SENSE (CS-SENSE) [21, 22] was performed a posteriori prior to reconstruction, and its effect on the reconstructed metabolite distributions was analyzed using both simulated datasets and those from healthy volunteers.

\section{THEORY}

\section{A. Experimental framework}

Considering MRSI data measured by phased array coils, MRSI signal measured by coil element $c=$ $1, \ldots, N^{c}$ at time $t$ and at Fourier-space coordinate $\mathbf{k}$ can be expressed as

$$
s_{c}(\mathbf{k}, t)=\int_{\Omega \subset \mathbb{R}^{3}} \rho(\mathbf{r}, t) C_{c}(\mathbf{r}) \mathrm{e}^{-2 \pi \mathrm{i} t \Delta B_{0}(\mathbf{r})} \mathrm{e}^{-2 \pi \mathbf{i} \mathbf{k} \cdot \mathbf{r}} d \mathbf{r},
$$

with $\rho(\mathbf{r}, t)$ the local transverse magnetization, $C_{c}(\mathbf{r})$ the coil sensitivity profile and $\Delta B_{0}(\mathbf{r})$ the $B_{0}$ magnetic field inhomogeneity profile, $\Omega$ being the object spatial support. $\mathbf{k}=\frac{\gamma}{2 \pi} \int_{0}^{\tau} \mathbf{G}(t) d t$ is the Fourier-space (k-space) coordinate determined via application of a magnetic field gradient $\mathbf{G}(t)$ during time $\tau$ [23]. The aim of the MRSI data reconstruction is to retrieve $\rho(\mathbf{r}, t)$ from $s_{c}(\mathbf{k}, t)$ knowing $C_{c}(\mathbf{r})$ and $\Delta B_{0}(\mathbf{r})$. Practically, the observed MR signal is sampled on a discrete spatio-temporal grid. The acquired $\mathrm{k}$-space values are then represented as a set of vectors $\mathbf{k}_{i}$ with $i=1, \ldots, N^{k}$ and the acquired time samples as $t_{j}=\left(\frac{j-1}{S R}\right), j=1, \ldots, T$ with $S R$ the sampling rate in the chemical shift domain (defined in section III A). $C_{c}(\mathbf{r}), \Delta B_{0}(\mathbf{r})$ and the solution $\rho(\mathbf{r}, t)$ are also evaluated at discrete spatial coordinates $\mathbf{r}_{l}$ with $l=1, \ldots, N^{r}$, the latter utilizing the same temporal grid $t_{j}$.For 2D MRSI, slice selective excitation restricts the spatial acquisition and reconstruction domain to a twodimensional subspace so long as sufficiently thin slices are achieved to justifiably discount partial voluming effects. $\mathrm{K}$-space values are generally acquired on a Cartesian grid with $\Delta \mathbf{k}=\left(1 / F O V_{x}, 1 / F O V_{y}\right)$ with $F O V_{x, y}$ the size of the field of view along the $\mathrm{x}$ and $\mathrm{y}$ axes, respectively. The k-space grid is filled to different extends following specific patterns as described further (section IIIB). 2D spatial coordinates of the reconstructed dataset, $\mathbf{r}_{l}$, cover a full rectangular Cartesian grid within the selected slice with $\Delta \mathbf{r}=\left(F O V_{x} / N_{x}, F O V_{y} / N_{y}\right)$ with $N_{x, y}$ the spatial resolution along $x$ or $y$ axis. In contrast to [19], spatial resolution in this work was set to the Nyquist limit without superresolution reconstruction. The fully discretized (1) then reads

$$
s_{c, i, j}=\sum_{l=1}^{N^{r}} F_{i, l} C_{c, l} B_{l, j} \rho_{l, j}+\epsilon_{c, i, j},
$$

with $B_{l, j}=\mathrm{e}^{-2 \pi \mathrm{i} t_{j} \Delta B_{0_{l}}}$ and $F_{i, l}=\mathrm{e}^{-2 \pi \mathrm{i} \mathbf{k}_{i} \cdot \mathbf{r}_{l}}$ and $\epsilon_{c, i, j}$ additive measurement noise assumed to be Gaussian. For sake of brevity, (2) can be expressed in a vector and operator form as

$$
\mathbf{s}=\mathcal{F C B} \boldsymbol{\rho}+\epsilon
$$

with $\mathcal{B}$, applying frequency shift due to $B_{0}$ inhomogeneity, $\mathcal{C}$, applying spatial coil sensitivity profiles and $\mathcal{F}$, the Fourier transform operator.

\section{B. Lipid suppression using orthogonality}

Bilgic et al. previously demonstrated effective removal of contaminating lipid signals by exploiting metabolitelipid spectral orthogonality $[18,24,25]$. As an illustration, let us define two complex time series $f_{j}^{M}, f_{j}^{L}$ $(j=1, \ldots, T)$ that contain signal from brain metabolites and skull lipids, respectively. The spectral orthogonality property reads

$$
\sum_{j=1}^{N^{t}} \overline{f_{j}^{M}} f_{j}^{L}=0
$$

where the over-line is the complex-conjugate. This property was verified experimentally in vivo [25]. MR time series acquired with $T$ time samples (e.g. $\left(s_{c, i, 1}, s_{c, i, 2}, \ldots, s_{c, i, T}\right)$ in (2) for any coil $c$ and $\left.\mathbf{k}_{i}\right)$ are elements of the complex vector space $\mathbb{C}^{T}$. Let $S^{M}$ and $S^{L}$ be two orthogonal subspaces of $\mathbb{C}^{T}$ that respectively contain metabolite and lipid MR times series. We the define the lipid subspace projection $\mathbf{P}$ such that $\mathbf{P f} \in S^{L}$ with $\mathbf{f}$ a general spectrum combining lipid and metabolite signals: $\mathbf{f}=\alpha \mathbf{f}^{M}+\beta \mathbf{f}^{L}, \mathbf{f}^{M} \in S^{M}, \mathbf{f}^{L} \in S^{L}, \alpha, \beta \in \mathbb{C}$. Because lipid and metabolite time series are orthogonal (following (4)), we have $\mathbf{P f}^{M}=0$ and the metabolite time series can be retrieved from $\mathbf{f}$ by

$$
(\mathbb{1}-\mathbf{P}) \cdot \mathbf{f}=\alpha \mathbf{f}^{M},
$$

$\mathbb{1}$ being the identity operator. Let us consider now a spatio-temporal MRSI dataset contaminated by skull lipids and acquired in vivo as defined in (2). The signal originating from each coil element is distinct by a phase offset and a specific spatial sensitivity profile, intimating that lipid suppression should be performed on each 
coil element individually. With $\mathbf{s}_{c} \in \mathbb{C}^{N^{k} \times T}$ the MRSI dataset measured by coil element $c$, lipid-free MRSI data $\mathbf{s}_{c}^{\mathrm{LipFree}}$ are then retrieved by

$$
\left(\mathbb{1}-\tilde{\mathbf{P}}^{c}\right) \mathbf{s}_{c}=\mathbf{s}_{c}^{\text {LipFree }},
$$

with $\tilde{\mathbf{P}}^{c}=\mathbb{1}_{\mathbb{C}^{N^{k}}} \otimes \mathbf{P}^{c}$, the broadcasted projection onto $\mathbb{C}^{N^{k}} \otimes S^{L}$ with $\otimes$ the tensor product. The overall sensitivity and specificity of the method ultimately hinges upon a precise determination of $\mathbf{P}^{c}$ to avoid inefficient lipid suppression or unwanted removal of metabolite signal. One such $\mathbf{P}^{c}$ can be constructed from MRSI data located at the skull using the singular value decomposition (SVD):

$$
\begin{aligned}
\rho_{c^{\prime}, l, j} & =\sum_{i=1}^{N^{k}} \overline{F_{i, l}} s_{c^{\prime}, i, j}, \\
\mathbf{V} \boldsymbol{\Sigma} \mathbf{U}^{H} & =\left\{\rho_{c^{\prime}, l, j} \mid c^{\prime}=c, \mathbf{r}_{l} \in \Omega_{S k u l l}\right\}, \\
P_{i, j}^{c} & =\sum_{n=1}^{K_{L}} V_{i, n} V_{n, j}^{*}, i, j=1 \ldots T,
\end{aligned}
$$

with $\Omega_{\text {Skull }} \subset \mathbb{R}^{2}$ the skull lipids spatial support and ${ }^{H}$ the Hermitian conjugate. $\boldsymbol{\Sigma}, \mathbf{V}$ and $\mathbf{U}$ are the singular values, the left and the right-singular vectors respectively. $\mathbf{P}^{c}$ is a $T$-by- $T$ matrix defined by the linear combination of the first $K_{L}$ right-singular vectors of $\mathbf{V}$ that form an orthogonal basis for the approximated lipid subspace. The rank $K_{L}$ is determined by the brain-skull lipid energy balance as follows. The remaining lipid energy density after lipid suppression over spatial support $\Omega$ is $E_{\Omega}=\frac{1}{V_{\Omega}} \sum_{\mathbf{r}_{l} \in \Omega} \sum_{\omega_{m}=0_{p p m}}^{3_{p p m}}\left|\tilde{\rho}_{c, l, m}^{\text {LipFree }}\right|^{2}$ with $\tilde{\rho}_{c, l, m}^{\text {LipFree }}=$ $\sum_{i=1}^{N^{k}}\left(F_{i, l}\right)^{*} \sum_{j=1}^{T} \mathrm{e}^{-2 \pi \mathrm{i} t_{j} \omega_{m}} s_{c, i, j}^{\text {LipFree }}$, the spatial and temporal Fourier transform of the lipid-free MRSI dataset (6) with $\omega_{m}=S R(m / T-1 / 2)(m=1 \ldots, T)$, the discrete frequency values. The lipid suppression is considered sufficient if the remaining lipid energy density in the skull is equivalent to that in brain regions in the resulting MRSI datasets. This condition translates to finding the minimum $K_{L}$ such that $\frac{E_{\Omega_{\text {Skull }}}}{E_{\Omega_{\text {Brain }}}} \leq 1$ with $\Omega_{\text {Brain }}$ the spatial support of the brain tissues. In practice, the lipid suppression is repeated while incrementing $K_{L}$ until $\frac{E_{\Omega_{\text {Skull }}}}{E_{\Omega_{\text {Brain }}}} \leq 1$. The use of this criterion enables adaptive lipid suppression for each coil element. This metric is based on the notion that brain metabolites are contaminated by skull lipids because of the discrepancies in signal intensity. If the lipid signal at the skull was reduced to a level equivalent to that of the metabolite signal (i.e. $\frac{E_{\Omega_{\text {Skull }}}}{E_{\Omega_{\text {Brain }}}} \leq 1$ ), lipid contamination through the point-spread-function in brain tissues would become negligible.

The method presented here is based on the regularization model proposed by Bilgic et al. [18, 25] but necessitates less computation time. Indeed, the $\ell_{2}$-regularization requires the computation of a matrix inverse with $\mathcal{O}\left((T)^{3}\right)$ computational complexity, while the SVD-based method presented above requires $\mathcal{O}\left(K_{L}(T)^{2}\right)$. Furthermore, in the adaptive iterative process of lipid suppression presented above, SVD and $\mathbf{V}$ are computed once per coil element. The computation time is significantly reduced in comparison with an adaptive iterations approach using Bilgic's method where varying the regularization parameter would entail a full matrix inversion at each iteration.

The lipid suppression in eq. (6) is performed in acquired $\mathbf{k}_{i}$ and therefore is still valid when MRSI dataset are undersampled in $\mathrm{k}$-space (as described below in section IIIB). For the computation of $\mathbf{P}^{c}$, the discrete Fourier transform in (7) is performed only on the acquired $\mathbf{k}_{i}$ what is equivalent to fill un-acquired k-space points with zeros. Because the k-space undersampling is random (see Fig.1), this zero-filling creates incoherent spatial interference [26] similar to an increase of the noise. A consequence of this random aliasing is that voxels located at the skull contain the lipid signal plus aliased noise or metabolite signals originating from other part of the field of view. However, thanks to the much higher signal intensity of lipids relative to noise and metabolite signal, voxels in $\Omega_{\text {Skull }}$ contain sufficient lipid spectral quality for an accurate determination of $\mathbf{P}^{c}$.

\section{Low-Rank TGV Reconstruction}

The full MRSI dataset contains highly correlated measurements and is often assumed to be partially separable with a limited number of components $K[9,15,19,27]$. This low-rank approximation for the reconstructed MRSI data $\boldsymbol{\rho}$ reads (following notation in (2))

$$
\begin{aligned}
\rho_{l, j} & =\sum_{n=1}^{K} U_{l, n} V_{n, j}, \\
\text { or } \boldsymbol{\rho}= & \mathbf{U V}, \\
\text { where } & \mathbf{U} \in \mathbb{C}^{N^{r} \times K}, \mathbf{V} \in \mathbb{C}^{K \times T} .
\end{aligned}
$$

This space-time decomposition leads to factorization of the MRSI dataset into a finite set of characteristic time series $\mathbf{V}$ that are spatially distributed over the measured volume according to $\mathbf{U}$. Because the MRSI dataset is assumed to contain a finite number of distinct metabolite resonance frequencies that are independent of spatial location, the low-rank approximation is a suitable approximation. Moreover, noise contained in the dataset follows a random distribution in time and space and, therefore, cannot be represented by few characteristic spectra with specific spatial distribution. Accordingly, fitting of a low-rank model results in efficient denoising of MRSI datasets [9]. In addition to noise filtering effect, the separation of the MRSI dataset into spatial and temporal components (8) allows to apply specific constrains such as total generalized variation (TGV) [28, 29]. TGV regularization on spatial metabolite components permits effective denoising in space while preserving edges and 
avoiding stair-casing artifacts present in traditional firstorder total variation schemes. The low-rank approximation and TGV regularization are applied through a reconstruction model proposed in [19], which has been extended here to account for the sensitivity profiles. $\mathbf{U}$ and $\mathbf{V}$ are determined by the minimization problem:

$$
\begin{gathered}
\arg \min _{\mathbf{U}, \mathbf{V}}\|\mathbf{s}-\mathcal{F C B}\{\mathbf{U V}\}\|_{2}^{2}+\lambda \sum_{n=1}^{K} \operatorname{TGV}^{2}\left\{U_{n}\right\}, \\
\text { where } \operatorname{TGV}^{2}\left\{U_{n}\right\}= \\
\arg \min _{\mathbf{A}}\left\|\nabla U_{n}-\mathbf{A}\right\|_{1}+\frac{1}{4}\|\mathcal{E}(\mathbf{A})\|_{1},
\end{gathered}
$$

with $\nabla$ and $\mathcal{E}$ being first and second order derivative operators (more details given in [28]) and $\mathbf{A} \in \mathbb{C}^{N^{r} \times 2}$ an auxiliary vector field. Since the TGV penalty enforces the spatial components to be sparse in finite absolute differences and because the coil sensitivity information is included, the model allows for CS-SENSE acceleration $[21,22,26,30]$ if $\mathrm{k}$-space is sparsely and randomly sampled. As the temporal signal is always fully sampled and effectively denoised by the low-rank constraint, no further regularization was performed on $\mathbf{V}$. Also the absence of additional constraint on time components permit to preserve original temporal characteristics. The reconstruction described by the optimization problem in (9) was carried out following procedure described fully in [19]. In short, to solve the low-rank TGV optimization problem, a primal-dual method is used for convergence of the spatial components in combination with projected gradient descent for the temporal components. Unfortunately, the full procedure for jointly estimating the spatial and spectral components is typically non-convex and can be time-consuming. To cope with these limitations, the initial estimates, $\mathbf{V}_{0}$ and $\mathbf{U}_{0}$, were determined through a singular value decomposition of the adjoint solution, which is presumed to lie close to the true inverse in the solution space. An SVD is then performed on the adjoint solution to compute initial conditions $(\mathcal{F C B})^{H} \mathbf{s}=\mathbf{U}_{\mathbf{0}} \mathbf{V}_{0}^{H}$ where $\mathbf{U}_{0}$ includes the left-singular vectors multiplied by the singular value matrix.

\section{METHODS}

\section{A. Sequence}

A slice selective high-resolution ${ }^{1} \mathrm{H}-\mathrm{FID}-\mathrm{MRSI}[2,3,6-$ 8] including a prior WET [31] water suppression (Fig. 1) was implemented on a $3 \mathrm{~T}$ Prisma fit MRI scanner (Siemens, Erlangen, Germany) using a receiver head coil with $N_{c}=64$ elements. The duration of phase-encoding and refocusing gradients were shortened to achieve an echo time (TE) of $1.1 \mathrm{~ms}$. The FID signal was acquired with $T=1024$ points at a $4 \mathrm{kHz}$ sampling rate followed by spoiler gradients with a repetition time (TR) of

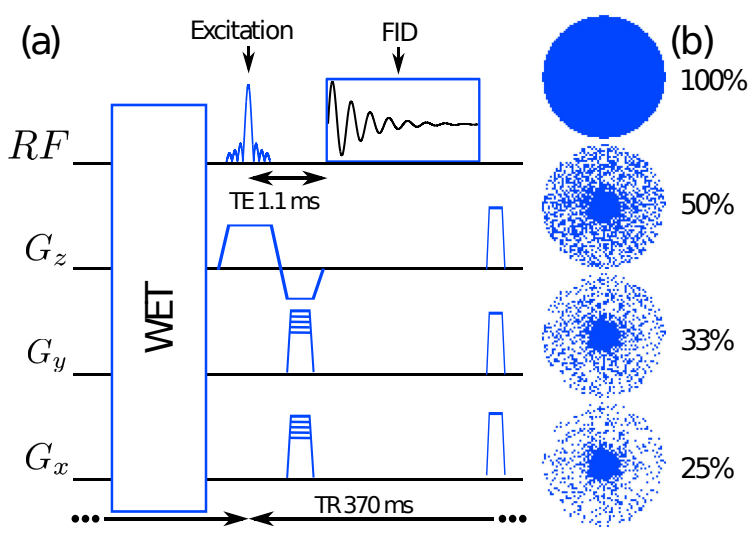

FIG. 1: (a) Diagram of the FID-MRSI sequence with radiofrequency signal $(R F)$ and gradients intensities along z,y and x directions $\left(G_{z}, G_{y}, G_{x}\right)$. (b) Illustration of the elliptical kspace sampling corresponding to acceleration factor $1,2,3,4$.

$370 \mathrm{~ms}$. Assuming that the maximum T1 value among metabolite is $1400 \mathrm{~ms}$ [32], the Ernst angle for the excitation pulse was computed to be 40 degrees. A smaller flip angle permits larger excitation pulse bandwidths while keeping within system voltage limits. A slice selective excitation pulse of $0.9 \mathrm{~ms}$ duration and $9.5 \mathrm{kHz}$ bandwidth was optimized with a Shinnar-LeRoux algorithm [33]. To determine the coil sensitivity profiles, two successive fast reference water acquisitions with head coil and body coil were added to the protocol. For this purpose, the same FID-MRSI sequence and encoding scheme was used but without WET and with a TR of $31 \mathrm{~ms}, 48$ FID sample points and a 5 degree flip angle.

\section{B. A Posteriori k-space Undersampling Acceleration}

Acquisition time can be significantly reduced by CSSENSE acceleration. For the 2D-MRSI acquisition scheme described above, acceleration is achieved through random $\mathrm{k}$-space undersampling in both phase encoding directions [26]. The acceleration factor (AF) is then the inverse of the $\mathrm{k}$-space filling. Here, an CS-SENSE acceleration was simulated a posteriori via variable density random removal of data points in $\mathrm{k}$-space. Nonuniform undersampling schemes have been shown to perform better than uniform patterns because low spatial frequencies carry most of the signal energy $[22,26]$. In practice, acquired k-space values $\boldsymbol{k}_{i}$ were removed randomly from $\mathbf{s}$ in (2) until reaching the desired AF with $i=1, \ldots, N^{k} / A F$ (after re-indexing). The random undersampling was constrained to result in a $1 /|\mathbf{k}|$ density distribution but with a fully-sampled center for $|\mathbf{k}| \leq$ $\frac{1}{8} \max (|\mathbf{k}|)=\frac{8}{220} \mathrm{~mm}^{-1}$ for a $220 \mathrm{~mm}$ square Field-ofView and $64 \times 64$ matrix size (Fig. 1 ). To meet real acceleration conditions, the random undersampling was per- 
formed on raw data prior to the data processing pipeline (incl. water suppression, lipid suppression and reconstruction). Lipid suppression (6) and the reconstruction model (9) remained the same but with a reduced size for $\mathbf{s} \in \mathbb{C}^{N^{c} \times \frac{N^{k}}{A F} \times T}$ and with a Fourier encoding operator $\mathcal{F}=\sum_{i=1}^{N^{k} / A F} F_{i, l}$.

\section{MRSI Data Processing and Reconstruction}

MRSI data acquired with full or undersampled k-space patterns were processed with the same pipeline described above and illustrated in the visual summary shown in Supporting Information Fig.1. First, residual water in MRSI data $\mathbf{s}$ was removed for each coil element and k-space position separately using Hankel singular value decomposition (HSVD) method [34]. Then, $\mathbf{s}_{c}$ the signal of each coil element was decontaminated from skull lipids using metabolite-lipid orthogonality (6). To determine the necessary $\Omega_{\text {Skull }}$ for the computation of $\mathbf{P}^{c}$, a mask was created by manual delineation of skull tissues on anatomical images (3D MPRAGE sequence). Coil sensitivity profiles were computed using data from the two fast water signal acquisitions. Because the signal measured by the body coil is presumed to be spatially homogeneous, the ratio of head coil element signal over body coil signal provided sensitivity profiles needed for the operator $\mathcal{C}$. Operator $\mathcal{B}$ was estimated using the multiple signal classification algorithm (MUSIC) [35] on the combined water signal from all the head coil elements. The spatio-temporal magnetization, $\boldsymbol{\rho}$, was then reconstructed from lipid-free MRSI data $\mathbf{s}_{c}^{\text {LipFree }}$ with the lowrank TGV model (9) using aforementioned operators $\mathcal{C}$ and $\mathcal{B} . \quad \rho$ become the input for the quantification step described below.

\section{LCModel Quantification}

The MRSI dataset $s(\mathbf{r}, t)$ resulting from the reconstruction (Eq. 8) is eventually quantified using LCModel [36] to estimate metabolite distributions. As a reference basis for LCModel, metabolite spectra were simulated with the GAMMA package [37], at $3 \mathrm{~T}$ and with an acquisition delay corresponding to the aforementioned employed acquisition scheme. Due to the rather low SNR in each voxel, only high signal metabolites were included in the spectrum basis: N-acetylaspartate, $\mathrm{N}$-acetyl aspartylglutamate, creatine, phosphocreatine, phosphorylcholine, glycerophosphorylcholine, myo-inositol, scyllo-inositol, glutamate, glutamine, lactate, beta-glucose and alanine. As an output, we obtain the spatial distributions $\boldsymbol{D}^{M}$ of metabolite $M$ being: tNAA (N-acetylaspartate and $\mathrm{N}$-acetyl aspartylglutamate), tCr (Total creatine), Cho (Choline containing compounds),Ins (Myo-inositol), Glx (Glutamate and Glutamine). LCModel quantification estimates the Cramer-Rao Lower bound (CRLB) and SNR

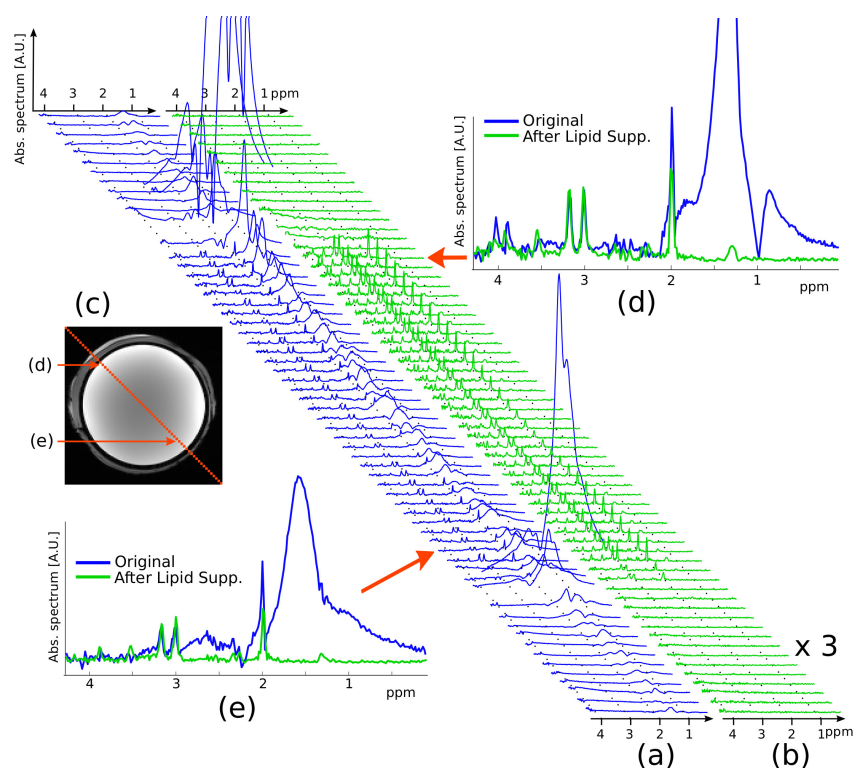

FIG. 2: Fat Phantom FID-MRSI content before (a) and after (b) lipid suppression by orthogonality. The 64 spectra originate from line of red voxels in the anatomical image (c). The contamination of the metabolite spectra inside the phantom is clearly visible in the original data (blue line) over the whole diagonal. The spectra after lipid suppression (green line) are magnified 3 times for ease of reading. Detailed spectra are shown on same scale in insets (d) and (e). They correspond to locations near the lipid layers shown by the red arrows. Contaminating lipid peaks are removed from the spectra over the whole phantom without affecting metabolite signal.

for each voxel [38] as spectral quality metrics. These outputs were then used to create CRLB and SNR maps for each measurement.

\section{E. Fat Phantom Experiment}

To assess lipid suppression performance, an in-house phantom was assembled to mimic a human head. To this end, MRS Braino phantom (GE Medical Systems, Milwaukee, WI, USA) containing an homogeneous metabolite solution (12.5 mM of N-acetyl-L-aspartic acid, $10 \mathrm{mM}$ of creatine hydrate, $3 \mathrm{mM}$ of choline chloride, $7.5 \mathrm{mM}$ of myo-inositol, $12.5 \mathrm{mM}$ of L-glutamic acid, $5 \mathrm{mM}$ of DLlactic acid, sodium azide $(0.1 \%), 50 \mathrm{mM}$ of potassium phosphate monobasic, $56 \mathrm{mM}$ of sodium hydroxide and $1 \mathrm{~mL} / \mathrm{L}$ of Gd-DPTA) was wrapped in a layer of hypodermic swine fat (Fig. 2). Prior to experimentation, the fresh fat layer $(<24 \mathrm{~h})$ was kept at $4^{\circ} \mathrm{C}$ to avoid lipid structure degradation. The phantom fat layer was wrapped as tight as possible to prevent air presence at the interface with Braino. MRSI data were acquired on a $10 \mathrm{~mm}$ slice with a $220 \times 220 \mathrm{~mm}$ Field-of-View (FoV) and $64 \times 64$ elliptical in-plane encoding. A structural volume for the delineation of the lipid layer was acquired with 


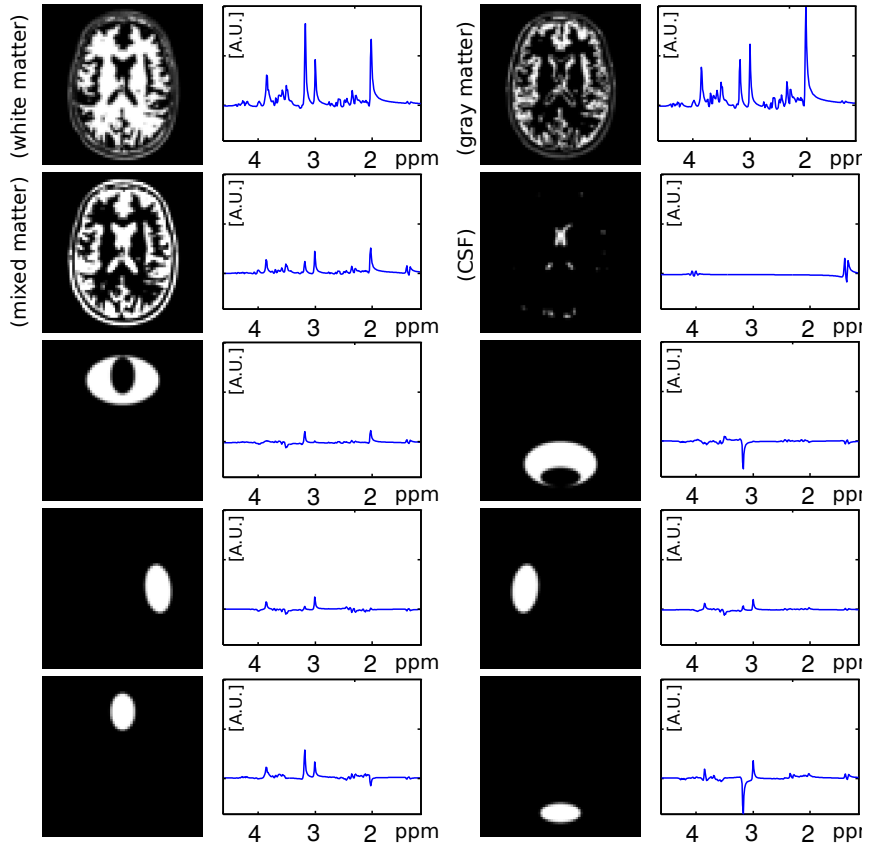

FIG. 3: The 10 compartments of the simulated MRSI analytical phantom are displayed next to their contained spectra. On each diagram is shown the real part of the spectrum that is a mix of N-acetylaspartate, creatine, glycerophosphorylcholine, myo-inositol, glutamate, glutamine and lactate. All 10 spatiospectral components are overlayed in the resulting dataset. The 4 top compartments are representative of a white matter, gray matter, mixed gray matter-cerebrospinal fluid (CSF) and CSF. The 6 lower elliptic compartments mimic small local modifications in metabolite concentrations.

a 3D T1-weighted MPRAGE sequence. After residual water removal, lipid contamination was removed using the method described above. Resulting lipid-free data from all coil elements were recombined using Roemer's weights [39] and quantified using LCModel to estimate concentration distributions $\boldsymbol{D}^{M}$ following the aforementioned notations (see No Model Pipeline in Supporting Information Figure 1). For comparison, the same processing pipeline was applied but without lipid suppression. The No Model Pipeline was chosen to highlight the efficacy of the lipid suppression in its own right, independent of the reconstruction.

\section{F. Simulated Data Experiment}

To benchmark the reconstruction using the low-rank TGV model, simulated MRSI dataset were produced with an analytical phantom [40] containing 10 compartments (8). The phantom represents an ideal and unbiased framework for assessing reconstruction performance, as it can be expressed in an analytical form in both image space and k-space. To approximate realistic experimental conditions, a $B_{0}$-induced frequency shift was added to the simulated data. This shift was added as a final step, as the introduction of a space dependent frequency shift precludes analytical solutions to the forward imaging equation. The 10 compartments comprising the phantom were created out of ellipses and Bézier curves (Fig. 4). Each compartment was associated with a time series containing simulated resonances (1024 pt at $4 \mathrm{kHz}$ with GAMMA package [37]) corresponding to N-acetylaspartate, creatine, glycerophosphorylcholine, myo-inositol, glutamate, glutamine and lactate (Fig. 3). The k-space MRSI data from 16 coils (positioned in a circular fashion around the analytical phantom with BiotSavart sensitivity profiles) was calculated for a $64 \times 64$ elliptical encoding matrix. MRSI data were then frequency shifted in accordance with a random $B_{0}$ field map constructed by filling a $8 \times 8$ with random frequencies taken from a Gaussian distribution $\mathcal{N}(\mu=0 \mathrm{~Hz}, \sigma=10 \mathrm{~Hz})$ and then spline interpolating up to the full $64 \times 64$ spatial grid. The resulting $B_{0}$ field map (Fig. 4) exemplifies the spatial smoothness and frequency range typically found in vivo. Eventually, random Gaussian noise was added to the simulated MRSI dataset with varying amplitude to obtain a desired SNR (defined by the ratio of the signal and noise standard deviations). The Matlab (The MathWorks, Inc., Natick, Massachusetts, US) scripts generating simulated MRSI dataset are available online [41] A spatio-temporal magnetization dataset $\rho^{\text {recon }}$ was reconstructed with the low-rank TGV model (9) and was compared with the analytical phantom solution in image space $\boldsymbol{\rho}^{\text {exact }}$. The performance of the reconstruction was assessed by a normalized root mean square error

$$
\mathrm{NRMSE}=\sqrt{\frac{\sum_{l, j}\left|\rho_{l, j}^{\text {exact }}-\rho_{l, j}^{\text {recon }}\right|^{2}}{\sum_{l, j}\left|\rho_{l, j}^{\text {exact }}\right|^{2}}}
$$

where $l, j$ span the spatial and temporal dimension (notation in section II A). Three batches of tests were performed on the model: 1) varying the regularization parameter $\lambda$ in (9) for SNR values $=1,2,4$ for $K=20$ components (8). 2) varying the number of component $K$ from 2 to 128 with optimal $\lambda$ and $\mathrm{SNR}=2$. 3) varying $\lambda$ for $\mathrm{AF}=2,3,4,6$ with $\mathrm{SNR}=2$ and $K=20$ model components. The results of the tests were assessed with the NRMSE. To contextualize reconstruction accuracy, the performance of the low-rank TGV model was contrasted with that of other models proposed in the literature. As the first alternative model, $\mathrm{Wu}$ et al. proposed the same model but with fully static temporal components $\mathbf{V}$ [20]. In this case $\mathbf{V}$ is computed during reconstruction initialization by SVD of the Casorati matrix representing the $B_{0}$ corrected MRSI dataset[11]. $\mathbf{V}$ is then kept constant during the reconstruction procedure, in contrast with the scheme presented here (9) where $\mathbf{V}$ and $\mathbf{U}$ converge simultaneously. As the second alternative model, TGV was replaced by first-order total variation (TV) regularization in (9) with $\operatorname{TV}\left\{U_{c}\right\}=\left\|\nabla U_{c}\right\|_{1}$. As the third and last alternative model, the low-rank approximation was removed from the reconstruction. (9) then becomes 


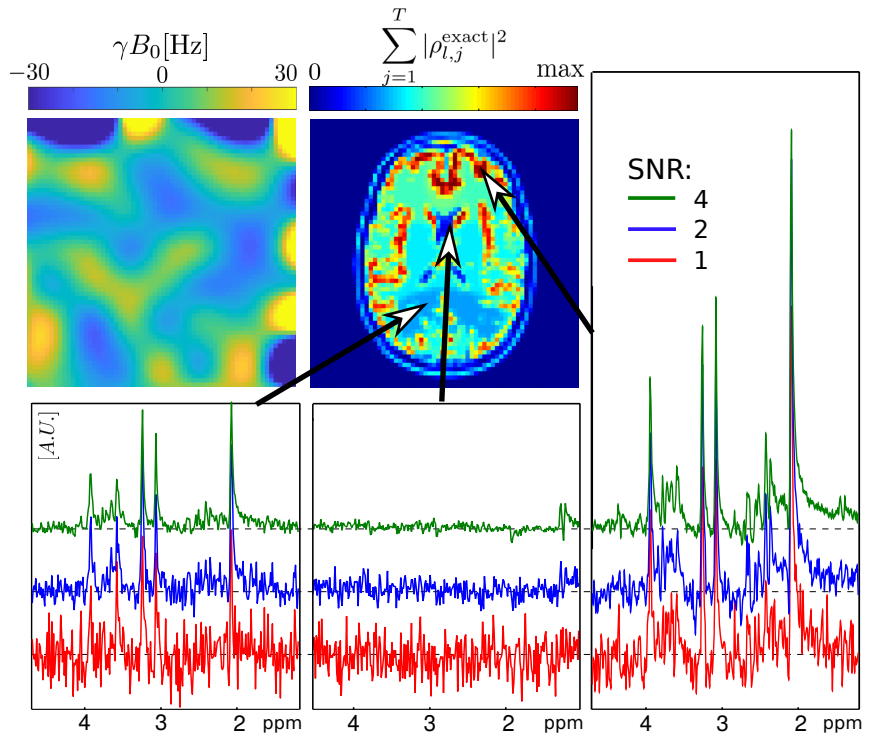

FIG. 4: Top, $B_{0}$ field inhomogeneity map and energy image of the analytical phantom in real space. The phantom is the combination of 10 overlay spectral compartments (Fig. 3). Bottom, spectrum real parts at 3 locations spotted by the arrows are shown with identical scale. the 3 colors correspond to 3 levels of noise added to the simulated dataset before the low-rank TGV model reconstruction.

$\arg \min _{\rho}\|\mathbf{s}-\mathcal{F C} \mathcal{B}\{\rho\}\|_{2}^{2}+\lambda \sum_{j=1}^{T} \mathrm{TGV}^{2}\left\{\rho_{j}\right\}$ (with notations from (2)). Reconstructions performed using each model were assessed with NRMSE in (10) with varying $\lambda$. Results were compared without acceleration or with $A F=2$ and $A F=4$.

\section{G. Healthy Brains Experiment}

MRSI Data from healthy volunteers were acquired with a protocol approved by the institutional ethics committee and written informed consent was given by all subjects before participation. Structural volume for positioning, was first acquired with a 3D T1-weighted MPRAGE sequence. Two FID-MRSI $10 \mathrm{~mm}$-thick slices were positioned axially at different locations. Elliptical $64 \times 64$ encoding over a FoV of $220 \times 220 \mathrm{~mm}$ resulted in a nominal voxel size of $3.4 \times 3.4 \times 10 \mathrm{~mm}$. A pair of fast water sequences were acquired (see III A) to create a reference signal for each slice. The total FID-MRSI data acquisition time was 21 min per slice (including water reference acquisitions). The resulting metabolite distributions $\boldsymbol{D}^{M}$ and spectral quality metric maps (CRLB and SNR) were computed for each subject acquisition. Results were compared to metabolite distributions reconstructed without the low-rank TGV modeling step. This no model pipeline consisted of HSVD water suppression, lipid suppression by orthogonality, coil signal combination using Roemer's method [39], inverse spatial Fourier transform and quantification with LCModel (sketch shown in Supporting In- formation Fig.1). A CS-SENSE acceleration was simulated a posteriori with $\mathrm{AF}=2,3,4$, and resulting accelerated $\boldsymbol{D}^{M}$ were compared to their fully-sampled counterparts.

\section{RESULTS}

\section{A. Fat Phantom Experiment}

Results of the lipid suppression method on the fat phantom are shown in Fig. 2 and Fig. 5. Spectra without lipid suppression displayed in Fig. 2.(a) show that the lipid signal originating from the fat layer contaminates the entire metabolite volume. Spectra from two voxels located at the fat layer are shown in Fig. 2.(c) and (d). After lipid suppression by orthogonality, results from the same voxels are displayed in Fig. 2.(e) and no noticeable lipid signal remains. The effect of the lipid suppression on metabolite distribution maps quantified by LCModel are displayed in Fig. 5 . The presence of lipid contamination in the MRSI dataset strongly alters the quantification of all metabolite concentrations and their ratios. When lipid suppression is applied beforehand, the quantification results reveal the actual homogeneous metabolite distributions. Metabolite distributions on the side of the phantom appear more intense due to the fact that coil element signals were combined with Roemer's method [39] without correction for their sensitivity profiles. The two metabolite ratios exhibit constant values inside the phantom when lipid suppression is applied.

\section{B. Simulated Data Experiment}

Results on the analytical phantom with different noise levels are presented in Fig. 6 top. In the low regularization regime $\left(\lambda \leq 10^{-4}\right)$, the error in the reconstructed dataset is influenced by the noise level present in the original dataset and decreases for greater $\lambda$. The minimal error is achieved within the optimal regularization parameter range $\left(5 \cdot 10^{-4} \leq \lambda \leq 10^{-3}\right)$ and NRMSE minimum values are nearly the same for the 3 SNR levels. For $\lambda \geq 10^{-2}$, the reconstruction produces over-regularized solutions that are nearly independent of $\lambda$. The reconstructed solution is not affected markedly by the number of components $K$ for $K \geq 8$ (Fig. 6 inset). When $K<6$, the model lacks the number of components to fit the data. However, for $K>32$ the model absorbs measurement noise into superfluous components, increasing NMRSE accordingly. Qualitative observation of spectral and spatial components, $V_{n}, U_{n}$, with $n>20$ reveals mainly noise (not shown here). For comparison, NRMSE values of the analytical phantom data reconstructed using a simple inverse Fourier transform are 0.378, 0.27, 0.216 for SNRs of 1,2 and 4 respectively. Simulated data reconstruction using the TGV low-rank model allowed therefore to reduce the error roughly tenfold using the optimal regularization 


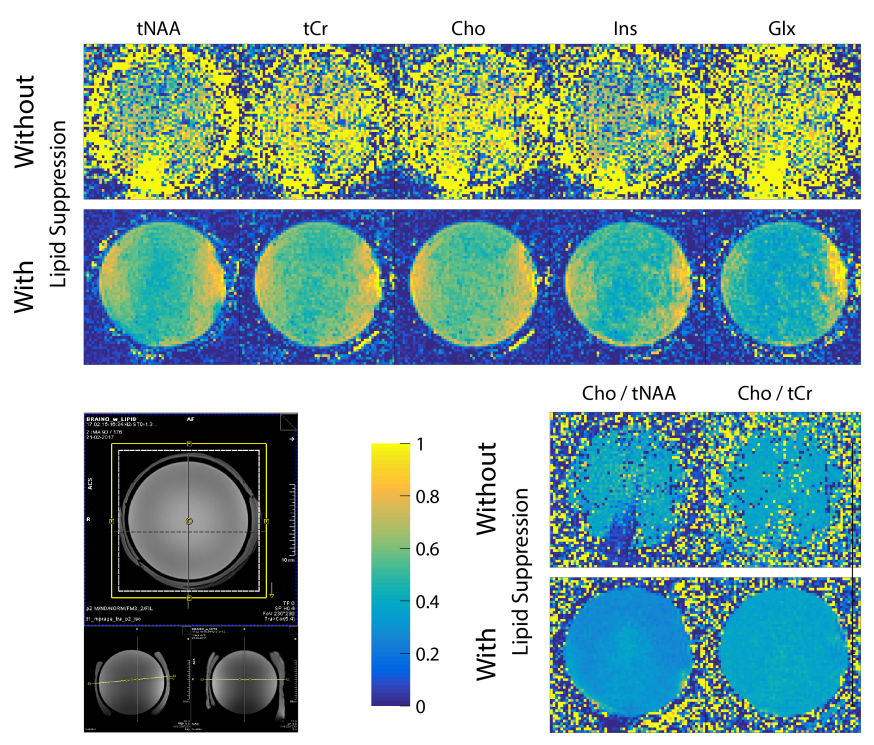

FIG. 5: Results of the fat phantom experiment. On top, the concentration distributions estimated by LCModel with or without the preceding lipid suppression step. The scale of each metabolite image with lipid suppression ranges from 0 to the 99th data percentile. The identical scale was then applied to the same metabolite image without lipid suppression. Bottom right, metabolite ratio with or without lipid suppression are displayed with scale from 0 to 1 . Bottom left, the MPRAGE image of the fat phantom with the MRSI FoV (yellow square) are shown.

parameter, and fivefold in the under-regularized regime compared to inverse Fourier transform. Sample spectra reconstructed with the low-rank TGV model compared to the exact solution are presented in Supporting Information Fig.6.

Results from simulated data with acceleration are presented in Fig. 6, bottom. The minimum NRMSE is achieved around $\lambda=5 \cdot 10^{-4}$. In this optimal regularization region, the NRMSE increases with AF as expected. Energy maps (Fig. 6, right) exhibit the same trend with results converging closer to the analytical solution when the acceleration factor decreases. When $\lambda>5 \cdot 10^{-3}$, the model converges to over-regularized solutions as illustrated by the corresponding energy maps. For small regularization values $\left(\lambda \leq 10^{-5}\right)$, spatial regularization almost vanishes from the model reconstruction, yet random aliasing resulting from the k-space undersampling is still minimized by coil sensitivity profiles included in the reconstruction model (9). This regime corresponds to a SENSE-like acceleration with NRMSE minimized for $\mathrm{AF}=2$. This particular result is also illustrated by the energy maps that better coincide with the analytical solution when $\mathrm{AF}=2$. An explanation can be found by observing the presence of Gibbs ringing artifacts in the fully sampled results. When k-space is undersampled $(\mathrm{AF}>1)$, the spatial distribution is smoothed, thereby reducing the artifacts and hence the NRMSE. Gibbs ring- ing artifacts are successfully removed by the TGV regularization for $\lambda \geq 10^{-4}$. Reconstruction results from different models are presented in Fig. 7. Without acceleration, Wu's reconstruction with pre-determined $\mathbf{V}$ offers the lowest error for all $\lambda$ values. The low-rank TGV reconstruction and the low-rank TV reconstruction produce similar results with slightly higher error than Wu's reconstruction. The model including TGV but not the low-rank condition shows the largest error for all $\lambda$ values. For $\mathrm{AF}=2$, the low-rank TGV reconstruction and the low-rank TV reconstruction show the lowest NMRSE followed by Wu's and TGV no-low-rank reconstructions. With $\mathrm{AF}=4$, results follow the same trend but with larger NMRSE discrepancies methods. Overall, with or without acceleration, all methods produce minimum error in the same range $10^{-4} \leq \lambda \leq 10^{-3}$. Processing time was $40 \mathrm{~min}$ for Wu's method, $80 \mathrm{~min}$ for low-rank TV, $120 \mathrm{~min}$ for low-rank TGV and $180 \mathrm{~min}$ for the TGV nolow-rank method, independently of acceleration. Computations were performed in Matlab (Matlab2017a, The MathWorks, Inc., Natick, Massachusetts, US) on a workstation equipped with an $\operatorname{Intel}(\mathrm{R}) \mathrm{Xeon}(\mathrm{R}) \mathrm{E} 5-2620$ v3, $2.40 \mathrm{GHz} \mathrm{cpu}$ and 64 Gigabytes RAM memory.

\section{Healthy Brains Experiment}

Metabolite distributions measured in vivo and reconstructed with the low-rank TGV model are presented in Fig. 8. Each metabolite shows distinct concentration patterns that are common to the two subjects (and similar for the two additional subjects shown in Supporting Information Fig.2). The gray / white matter contrast is particularly evident in the $\mathrm{tCr}$, tNAA and Glx maps while Cho is present in high concentration through the frontal and cingulate white matter regions and in low concentration in the occipital lobe. The Ins distribution exhibits constant concentration throughout the whole brain. Metabolite datasets were quantified using an arbitrary institutional units scale that is identical for each metabolite (see LCModel documentation [38]). The effect of the low-rank TGV reconstruction step on the final metabolite distributions is shown in Fig. 9. A significant noise reduction is visible in the results with the lowrank TGV reconstruction, particularly in the maps of low signal metabolite: Cho, Ins and Glx. The Cramer-Rao Lower bound (CRLB) and SNR estimated by LCModel distinctly reflect this improvement with systematic lower CRLB and higher SNR in results from the model reconstruction. The distributions reconstructed without model suffer also from poor signal homogeneity as particularly visible on tNAA and $\mathrm{tCr}$ maps where the signal decreases with the distance to the coil elements. This inhomogeneity is corrected in the low-rank TGV reconstruction by the spatial coil sensitivity profiles $\mathcal{C}(9)$. Effect of the coil sensitivity correction in the model is also particularly visible in Fig. 9 on Cho which is a metabolite mainly present in white matter at the center of the brain. 


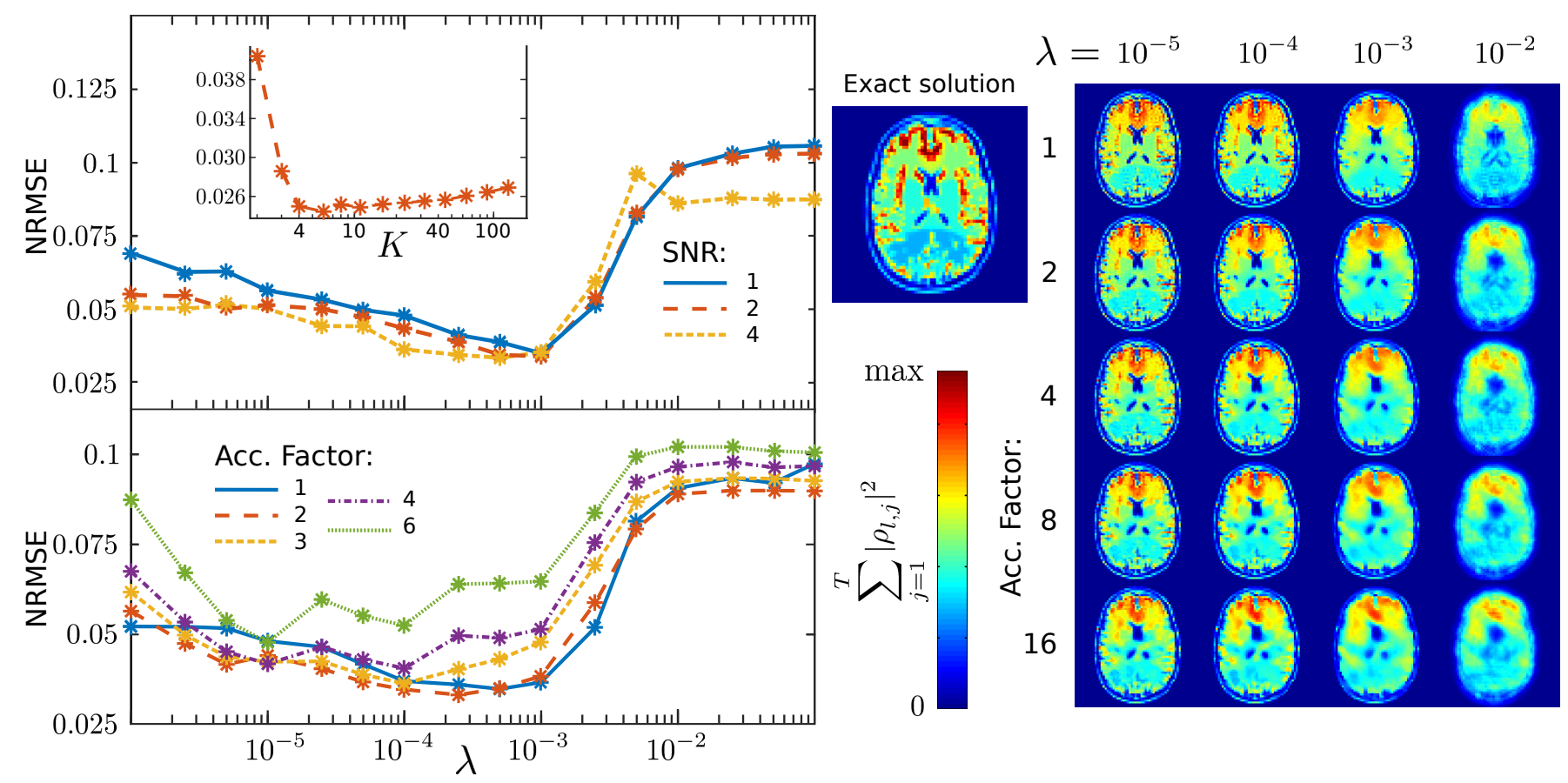

FIG. 6: Left, normalized root mean square error (NRMSE) of the reconstructed simulated MRSI data with respect to the analytical solution as function of the regularization parameter $\lambda(9)$. Top left, three datasets with different signal-to-noise ratio (SNR) are shown. Minimum NRMSE do not depend on SNR and is located at the same $\lambda$ values for all SNR. Top left inset shows NRMSE of the simulated MRSI dataset with $\mathrm{SNR}=2$ when reconstructed with $\lambda=5 \cdot 10^{-4}$ and different model rank $K(8)$. Bottom left, simulated data reconstruction with $\mathrm{SNR}=2$ as function of the regularization parameter $\lambda(9)$ and with different acceleration factors. Right, energy maps in real-space at targeted resolution of the exact analytical phantom (center) and of the model reconstruction (right) at several $\lambda$ values and acceleration factors.

In Fig. 10, the effect of CS-SENSE acceleration on the metabolite distribution maps is displayed. The patterns specific to each metabolite are preserved through the acceleration up to $\mathrm{AF}=4$ but the fine details of the maps are blurred.

To contrast performances for in vivo MRSI, reconstructions with the aforementioned models (presented in section III F) were performed on a healthy brain dataset. Results without acceleration and with $\mathrm{AF}=2,4$ are presented in Supporting Information Fig.3 . Resulting metabolite distributions $\boldsymbol{D}^{M}$ were compared qualitatively and show similar tendencies as the simulated data experiments. Without acceleration Wu's (static V), lowrank TGV and low-rank TV reconstructions produce similar metabolite distributions. TGV (no low-rank) reconstruction exhibits noisier metabolite distributions. With acceleration factor 2 and 4, greater differences between the methods are visible in the metabolite distributions. Low-rank TGV reconstruction seems to perform better than low-rank TV and Wu's reconstructions which show slightly greater distortions.

\section{DISCUSSION}

In this study, an original processing pipeline for $2 \mathrm{D}$ MR spectroscopic data acquired with a ${ }^{1} H$-FID-MRSI sequence is presented. The resulting reconstructed metabolic distribution maps are devoid of skull lipid contamination, and reveal increased spectral SNR and spatial fidelity. The reconstruction model was proven to be compatible CS-SENSE acceleration scheme. The effect of each pipeline step was analyzed and tested individually. The reconstruction method was compared to other alternative approaches proposed in the literature. The FID-MRSI sequence was implemented with a particular effort to reduce the echo-delay that causes 1st order phase distortion and $T_{2 *}$ signal loss. To this end, the refocusing gradient combined with the phase encoding gradients following the slice selective excitation pulse were made as short as possible within hardware limits. The metabolite signal acquired with $1.1 \mathrm{~ms}$ TE are highly quantitative without significant relaxation effects. The phase distortion present in the spectra was taken into account in the LCModel reference basis that was simulated with a FID sequence scheme and the utilized experimental TE. The fat phantom experiment was an attempt to reproduce the challenging measurement conditions encountered in vivo when MRSI data are acquired over a whole subject head including the skull fat layer. Without lipid suppression, metabolite signal from the brain would be thoroughly contaminated by the skull lipid signal as illustrated in Fig. 5 top. As mentioned by Bilgic et al. [25], the metabolite-lipid or- 


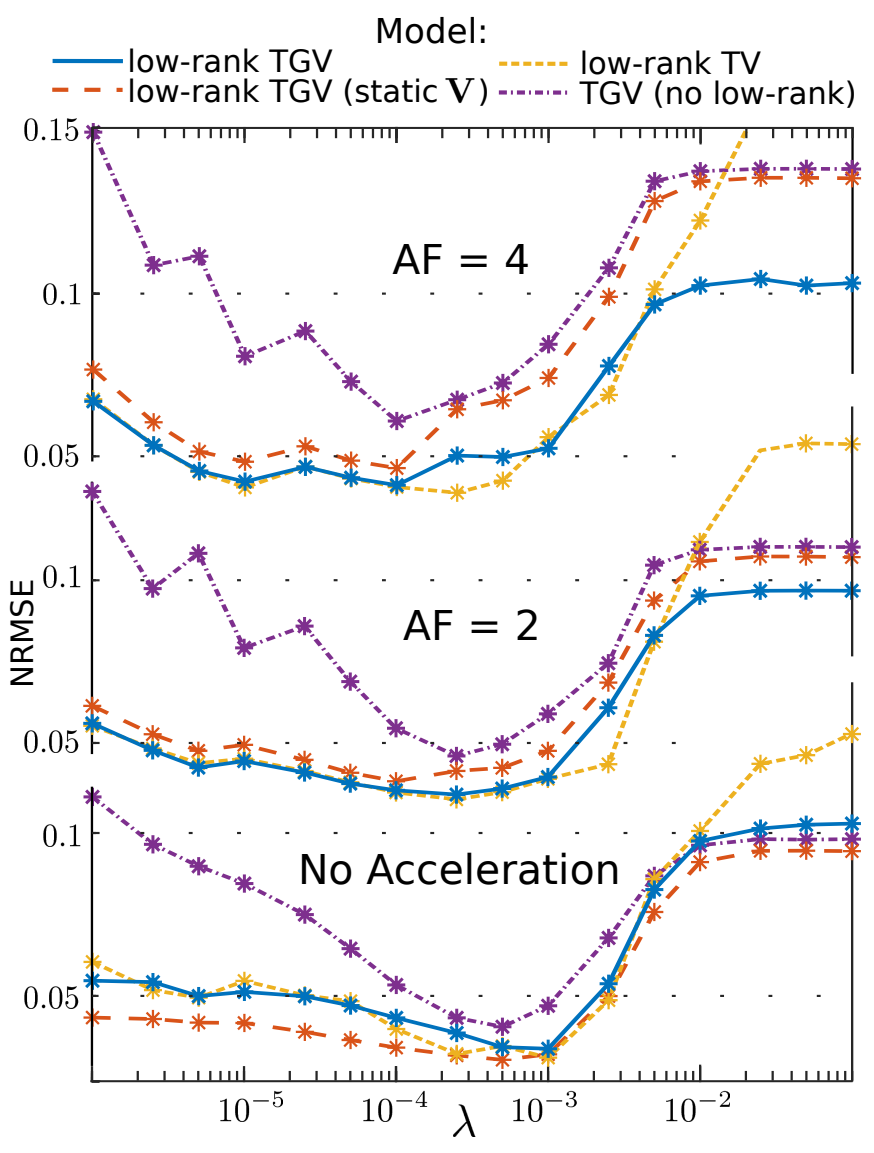

FIG. 7: Normalized root mean square error (NRMSE) of the simulated MRSI dataset with $\mathrm{SNR}=2$ as function of the regularization parameter $\lambda$ reconstructed with different models. Bottom, center and top curves correspond to data with no acceleration and acceleration factor (AF) 2 and 4 respectively. low-rank $T G V$ is the model used in this study (9), low-rank $T G V$ (static $\mathbf{V}$ ) is the same model but with pre-determined time components, low-rank $T V$ is identical but with total variation (TV) regularization and $T G V$ (no low-rank) is a reconstruction model including only TGV regularization without low-rank approximation.

thogonality approximation is not always valid. The positive results of the fat phantom experiment confirm nevertheless that this approximation is still sound for data acquired with ultra-short TE ${ }^{1} H$-FID-MRSI sequence. The lipid suppression method presented here is strongly based upon the method of Bilgic et al. [18, 25] which has been successfully utilized in a number of recent publications $[7,8]$. In [18], lipid suppression is expressed as an optimization problem that can be translated into the formalism of (6) with $\left(\nVdash-\boldsymbol{P}^{c}\right)=\left(\nVdash+\beta \boldsymbol{L} \boldsymbol{L}^{*}\right)^{-1}((12)$ in [18]). The advantage of the SVD-base approach is the reduced computational cost that allows for adjustment of lipid suppression strength via rank $K_{L}$ for each coil element in a reasonable computing time (approx. 10 minutes). The low-rank TGV regularized model used for reconstruction was shown to be effective in several re- gards. In the absence of acceleration, the model-based approach improved reconstruction accuracy over inverse Fourier as exhibited with the simulated data experiment (Fig. 6). For $\lambda=10^{-6}$, the effect of spatial regularization is effectively negligent, and the reconstruction reduces to a low-rank approximation [9]. In this case, NRMSE is reduced fourfold in comparison to spatial inverse Fourier transform for all SNR values on account of the implicit denoising. Reintroducing spatial regularization into the model with $\lambda$ set to optimal value of around $5 \cdot 10^{-4}$ achieve a tenfold reduction in NRMSE resulting in the lowest error when compared with ground truth data. Accordingly, reconstruction of the fully sampled in vivo MRSI dataset also results in improvements of precision as reflected by the CRLB and SNR, particularly for low signal metabolites (Fig. 9). Our analysis confirms previous reports (e.g.[19]) demonstrating that the low-rank TGV model significantly improves reconstruction accuracy over traditional inverse Fourier transform. CS-SENSE results of simulated MRSI datasets (Fig. 6) show that acquisition acceleration by random $\mathrm{k}$-space undersampling is possible without adversely affecting metabolite distributions when reconstructed with an optimal regularization parameter $\left(10^{-4} \leq \lambda \leq 10^{-3}\right)$ and with $\mathrm{AF} \leq 4$. Comparison between reconstruction methods shows that, in absence of acceleration, Wu's reconstruction performs slightly better than the low-rank TGV reconstruction used in this study, as illustrated by simulated data (Fig. 7). Reconstruction performance was also slightly better when using first-order total variation regularization. This is not an unexpected result, as the simulated phantom consists of exactly the type of piecewise constant geometries promoted by first-order total variation penalties. Under real-world experimental conditions, metabolite distributions from the acquired in vivo MRSI datasets exhibited similar quality between all methods without acceleration (Supporting Information Fig.3). However, when acceleration was used, superior reconstruction quality was achieved with the low-rank TGV-regularized method presented for both simulated and healthy volunteer MRSI data.

Inhomogeneities in the radio-frequency transmit field $\left(B_{1}^{+}\right)$may be a non-negligible source of error in quantitative MRI techniques due to the flip angle spatial inhomogeneity. However, with steady state magnetization following from the use of Ernst's flip angle, the effect of $B_{1}^{+}$deviation on the signal remains small. Indeed, letting single slice $B_{1}^{+}$deviation to represent a maximum $20 \%$ variation in the flip angle $[42,43]$, and with $T_{1}$ metabolite values ranging from 1000 to $1400 \mathrm{~ms}$ [32], steady state magnetization would be expected to change by only $5 \%$ at maximum ( see Supporting Information Fig.7). Low flip angle excitation enable the use of high bandwidth excitation pulse thereby minimizing chemical shift displacement error in the slice direction. For example, slice excitation of two chemical species separated by $500 \mathrm{~Hz}$ are shifted by $0.5 \mathrm{~mm}$.

As proof of concept, results on healthy volunteers il- 

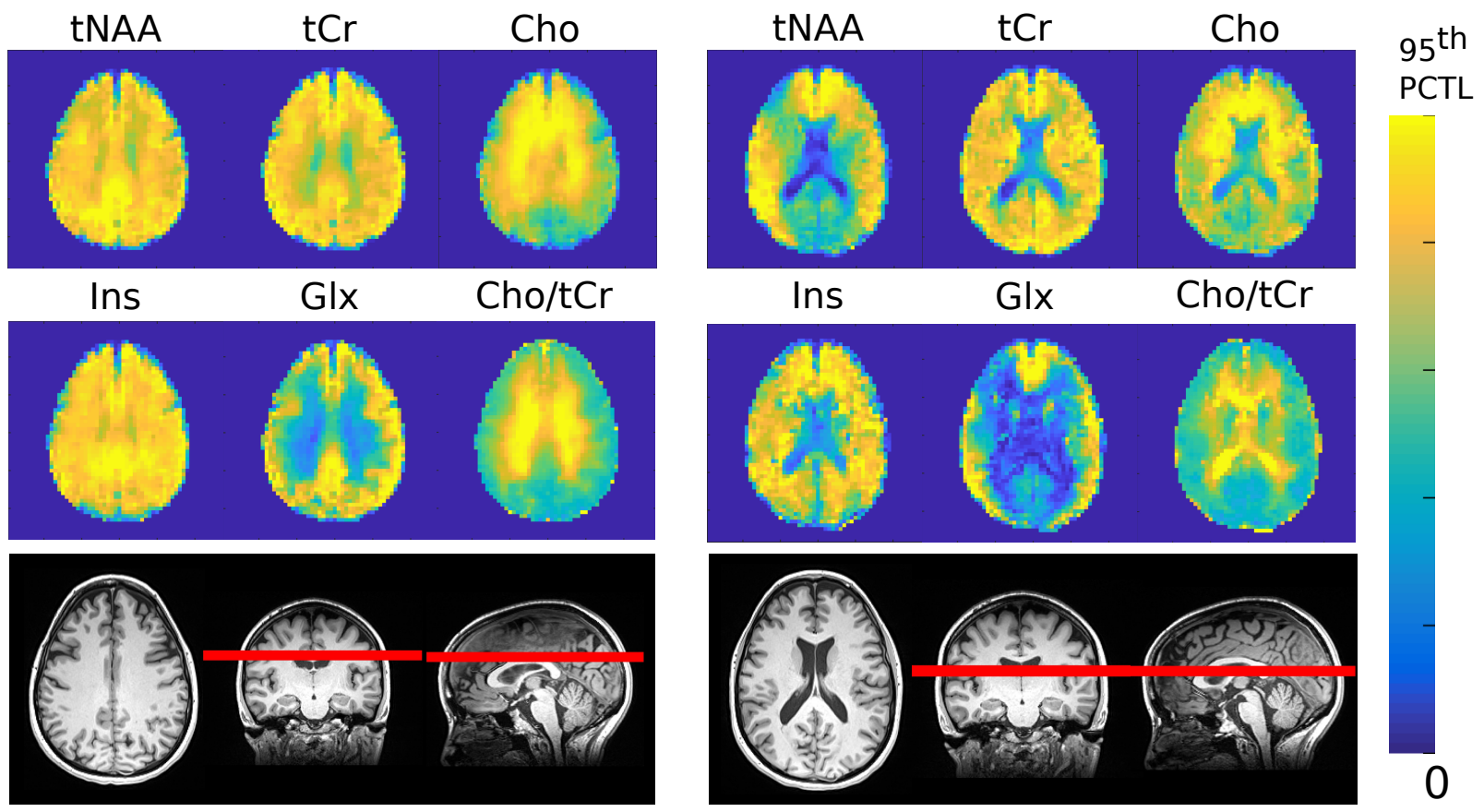

FIG. 8: Metabolite distributions $\boldsymbol{D}^{M}$ from two healthy volunteers reconstructed with the low-rank TGV model with optimal regularization parameter $\left(\lambda=10^{-3}\right)$ and rank $K=20$ without acceleration (two extra healthy volunteer results are shown in Supporting Information Fig.2). The maximum of the scale was set to the 95th percentile (PCT) of each metabolite image separately.

lustrate demonstrable improvement in SNR and CRLB, particularly for lower signal metabolites: Cho, Ins and Glx (Fig. 9). The reconstructed metabolite maps of two distinct volunteers as presented in Fig. 8 show the same metabolic features as previously published data at $7 \mathrm{~T}$ and higher field [7,8] affirming the in vivo reconstruction results. Consistency of the distribution across all volunteers for each metabolite demonstrates qualitative reproducibility of the methodology. Metabolite distributions are expressed in institutional units that are identical for each metabolite, thereby allowing comparison between cross-sectional or longitudinal subject data. Absolute metabolite quantification should therefore be possible using the reference water measurement if appropriate calibrations are done, and if the water signal is corrected for relaxation effects. However, water referencing might bias the estimated concentrations due to proton density variations between gray and white matter.

Results of in vivo metabolite maps accelerated with kspace undersampling (Fig. 10) in combination with the simulated data results (Fig. 6) indicate that the MRSI acquisition can be safely accelerated by CS-SENSE with $\mathrm{AF}=2$ without introducing significant quantitative and qualitative errors into the resulting metabolite distributions. This important license to clinicians to shorten the acquisition time of the 2D-FID-MRSI to 11 min per slice, and thereby bringing the technique in line with clinical requirements. The results presented in this article represent a proof of concept for the low-rank TGV reconstruction of 2D-FID-MRSI. We showed that the method- ology is effective in generating high-resolution metabolite distributions but further reproducibility tests should be performed in order to measure precision and robustness, and to assess results on slices positioned in different brain regions. Indeed, like for most MRSI sequences, the quality of a FID-MRSI dataset strongly depends upon the local $B_{0}$ homogeneity. Further investigation should be done to determine measurement feasibility in regions located near the temporal lobes or around hippocampi. Whole brain spectroscopic imaging is a topic gathering significant attention in the research community due to the original nature of the produced metabolite images and their possible applications in neurological and neuroscientific clinical research [44-46]. Numerous previous studies presented methodology based either on specific acquisition sequences [47, 48] and/or reconstruction techniques $[15,17,25,27,49]$. Spatial-spectral encoding and echo planar schemes have been shown to be fast and efficient for acquiring whole brain MRSI data in 3D, but at the cost of a lower SNR and longer TE [50-54]. Recent developments in FID-MRSI acquisitions at ultra high fields combined with acceleration techniques are particularly promising due to the high SNR and resolution of the resulting metabolite maps [2, 3, 6-8, 55-57]. The method presented in this article is an adaptation of the FID-MRSI approach for lower field strength. Although SNR is reduced by half compared to $7 \mathrm{~T}$, MRSI acquisition at $3 \mathrm{~T}$ benefits from improved $B_{1}$ and $B_{0}$ homogeneity, which may be particularly advantageous for FID-MRSI measurements in brain regions with less ho- 


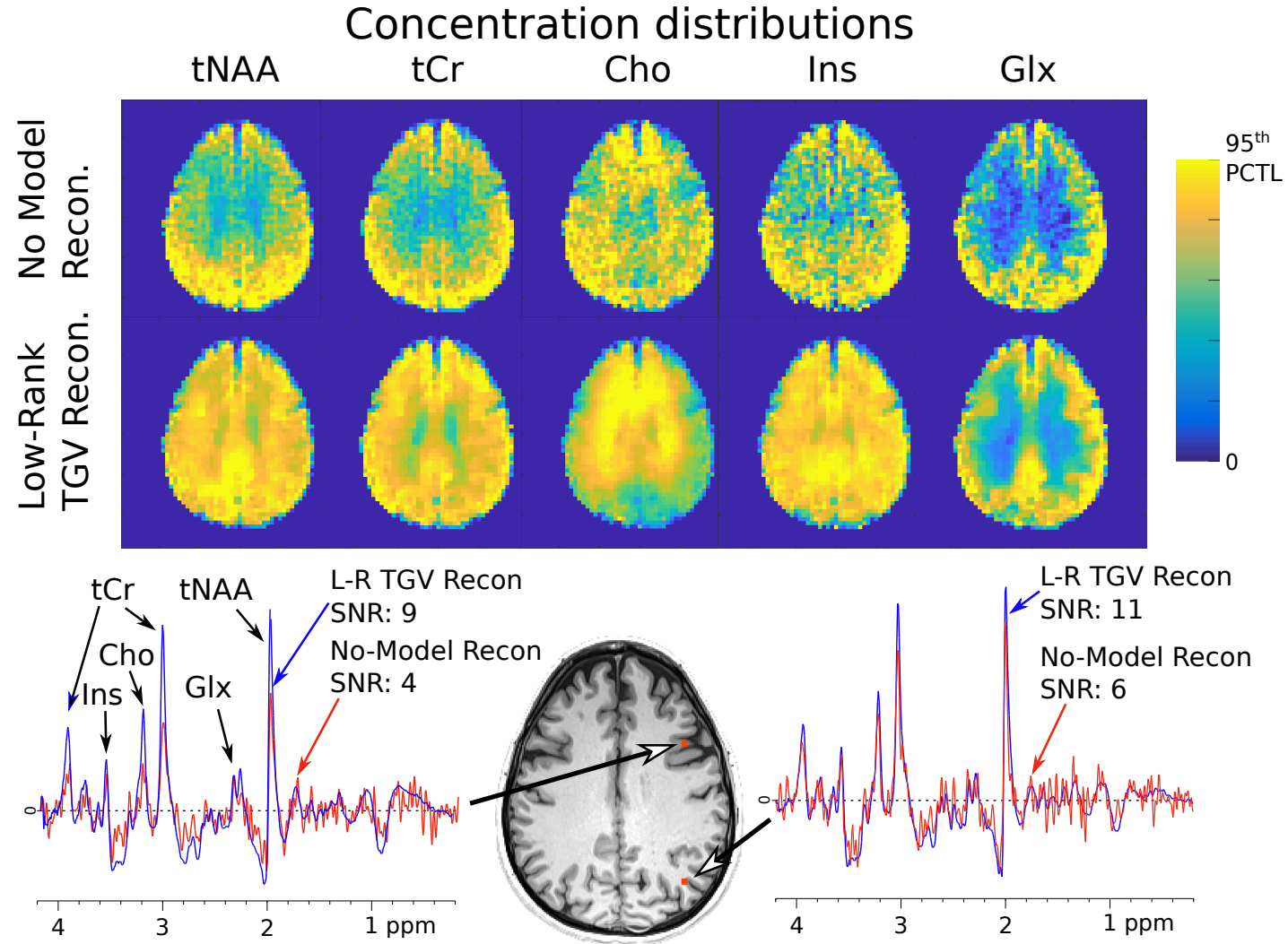

FIG. 9: Top, effect of the low-rank TGV reconstruction on the final metabolite distributions $\boldsymbol{D}^{M}$. For Low-Rank TGV Recon., distributions were reconstructed with the model and $\lambda=10^{-3}, K=20$. The metabolite distributions reconstructed without model (No Model Recon.) result from a pipeline including only lipid suppression and LCModel quantification. The scale ranges from 0 to the 95th percentile (PCTL) of each concentration distribution separately. Bottom, Two real parts of spectrum samples are shown with their respective locations. The red line belongs to the reconstruction without model and the blue line corresponds to the low-rank TGV reconstruction (Extended figure with CRLB and SNR maps in Supporting Information Fig.4).

mogeneous $B_{0}$. Also, new $B_{0}$-shimming methods with, for instance, dynamic multi-coil schemes [58] would certainly improve result quality in these conditions. To summarize, an original methodology for acquiring highresolution metabolite distributions was presented. The technique developed on a $3 \mathrm{~T}$ clinical MR system, requiring just 11 min with $\mathrm{AF}=2$ for acquisition of an entire $2 \mathrm{D}$ slice of the brain, should be of considerable interest for clinical research and applications.

\section{Acknowledgments}

This work was supported by the Centre for Biomedical Imaging (CIBM) of the University and University Hospitals of Geneva. The authors thank Mr. Jacky Bula and his employees for kindly providing free material for the fat phantom experiment.
[1] R. A. de Graaf, In Vivo NMR Spectroscopy: Principles and Techniques: 2nd Edition (2007), ISBN 9780470026700 .

[2] A. Henning, A. Fuchs, J. B. Murdoch, and P. Boesiger, NMR Biomed 22, 683 (2009).

[3] W. Bogner, S. Gruber, S. Trattnig, and M. Chmelík, NMR in Biomedicine 25, 873 (2012), ISSN 09523480.

[4] M. Považan, G. Hangel, B. Strasser, S. Gruber, M. Chmelík, S. Trattnig, and W. Bogner, NeuroImage 121, 126 (2015), ISSN 10538119.
[5] G. Hangel, B. Strasser, M. Považan, S. Gruber, M. Chmelík, M. Gajdošík, S. Trattnig, and W. Bogner, NMR in Biomedicine 28, 1413 (2015).

[6] G. L. Chadzynski, J. Bause, G. Shajan, R. Pohmann, K. Scheffler, and P. Ehses, Magnetic Resonance in Medicine 78, 1281 (2016).

[7] G. Hangel, B. Strasser, M. Považan, E. Heckova, L. Hingerl, R. Boubela, S. Gruber, S. Trattnig, and W. Bogner, NeuroImage 168, 199 (2018), ISSN 10538119 . 


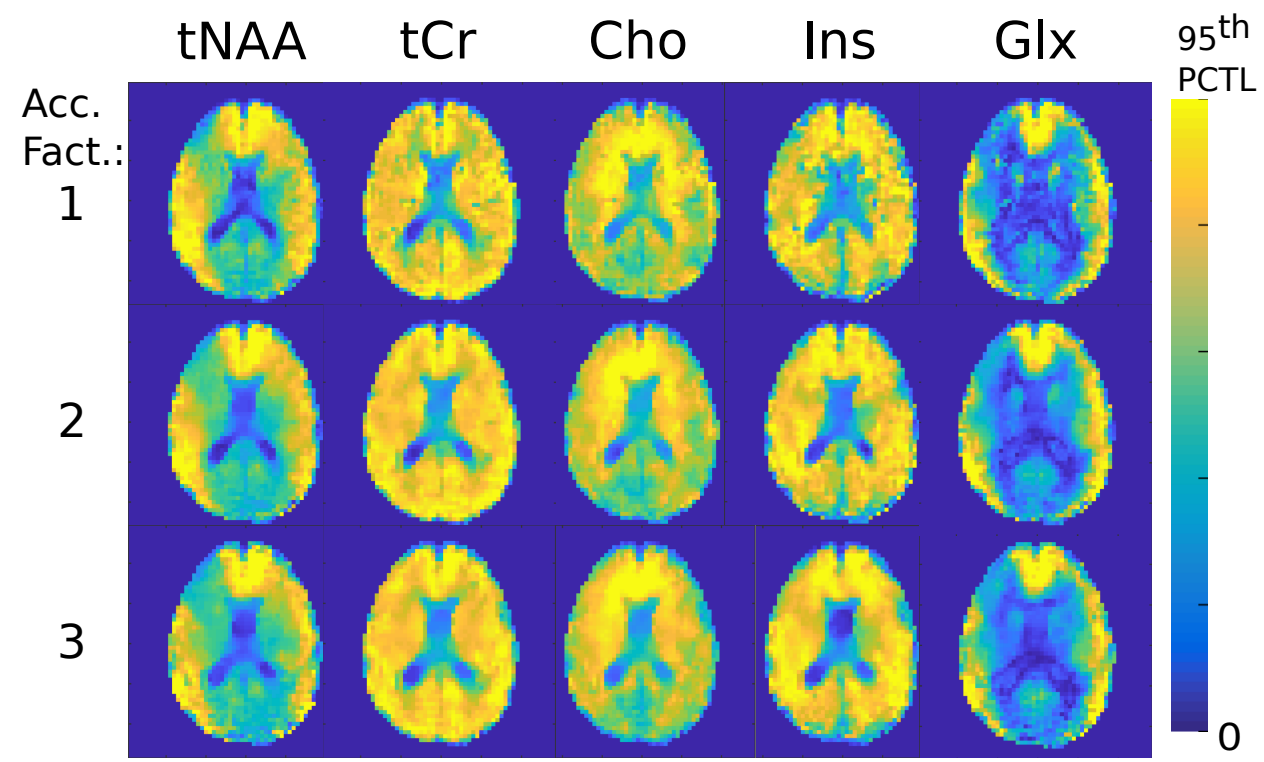

FIG. 10: Effect of k-space undersampling acceleration on metabolite distribution maps for an in vivo dataset. Reconstruction was performed with rank $K=20$, optimal regularization parameter $\left(\lambda=10^{-3}\right)$ and with acceleration factors $=1,2,3$. The scale ranges from 0 to the $95 \mathrm{th}$ percentile (PCTL) of each concentration distribution and acceleration factor separately (additional in vivo dataset and $\mathrm{AF}=4$ shown in Supporting Information Fig.5).

[8] S. Nassirpour, P. Chang, and A. Henning, NeuroImage 168, 211 (2018), ISSN 1053-8119.

[9] H. M. Nguyen, X. Peng, M. N. Do, and Z.-P. Liang, IEEE Trans Biomed Eng 60, 78 (2013).

[10] Y. Liu, C. Ma, B. Clifford, F. Lam, C. Johnson, and Z.$\mathrm{P}$. Liang, IEEE transactions on bio-medical engineering (2015), ISSN 1558-2531.

[11] F. Lam and Z. P. Liang, Magnetic Resonance in Medicine 71, 1349 (2014), ISSN 15222594, NIHMS150003.

[12] C. Ma, F. Lam, Q. Ning, C. L. Johnson, and Z.-P. Liang, Magnetic Resonance in Medicine 77, 467 (2017), ISSN 07403194.

[13] F. Lam, Y. Li, B. Clifford, and Z.-P. Liang, Magnetic Resonance in Medicine 79, 2460 (2018), ISSN 07403194.

[14] C. Ma, F. Lam, C. L. Johnson, and Z.-P. Liang, Magnetic Resonance in Medicine 75, 488 (2016), ISSN 07403194.

[15] I. Bhattacharya and M. Jacob, Magnetic Resonance in Medicine 78, 1267 (2016).

[16] C. Ma, B. Clifford, Y. Liu, Y. Gu, F. Lam, X. Yu, and Z.-P. Liang, Magnetic Resonance in Medicine 78, 419 (2017), ISSN 07403194.

[17] J. Kasten, A. Klauser, F. Lazeyras, and D. Van De Ville, J Magn Reson 263, 193 (2016).

[18] B. Bilgic, I. Chatnuntawech, A. P. Fan, K. Setsompop, S. F. Cauley, L. L. Wald, and E. Adalsteinsson, J Magn Reson Imaging 40, 181 (2014), ISSN 15222586, NIHMS150003.

[19] J. Kasten, F. Lazeyras, and D. Van De Ville, Medical Imaging, IEEE Transactions on 32, 1853 (2013).

[20] Zheng-Hua Wu, Fan Lam, Chao Ma, and Zhi-Pei Liang, in 2014 36th Annual International Conference of the IEEE Engineering in Medicine and Biology Society (IEEE, 2014), pp. 2432-2435, ISBN 978-1-4244-7929-0, ISSN 1557170X.

[21] D. Liang, B. Liu, J. Wang, and L. Ying, Magnetic resonance in medicine 62, 1574 (2009), ISSN 1522-2594.
[22] R. Otazo, D. Kim, L. Axel, and D. K. Sodickson, Magnetic Resonance in Medicine 64, 767 (2010), ISSN 07403194 .

[23] E. M. Haacke, R. W. Brown, M. R. Thompson, R. Venkatesan, M. A. Bernstein, K. F. King, and X. J. Zhou, Magnetic Resonance Imaging: Physical Principles and Sequence Design (2004), ISBN 0120928612.

[24] J. Lee and E. Adalsteinsson, in International Society for Magnetic Resonance in Medicine 19th Scientific Meeting (2010), p. 965.

[25] B. Bilgic, B. Gagoski, T. Kok, and E. Adalsteinsson, Magnetic Resonance in Medicine 69, 1501 (2013).

[26] L. Michael, D. David, and P. J. M., Magnetic Resonance in Medicine 58, 1182 (2007).

[27] F. Lam, C. Ma, B. Clifford, C. L. Johnson, and Z.-P. Liang, Magnetic Resonance in Medicine 76, 1059 (2016), ISSN 07403194, mrm.26019.

[28] K. Bredies, K. Kunisch, and T. Pock, SIAM Journal on Imaging Sciences 3(3), 492 (2010), ISSN 19364954.

[29] F. Knoll, K. Bredies, T. Pock, and R. Stollberger, Magnetic Resonance in Medicine 65, 480 (2011), ISSN 07403194

[30] K. P. Pruessmann, M. Weiger, P. Börnert, and P. Boesiger, Magnetic Resonance in Medicine 46, 638 (2001), ISSN 07403194.

[31] R. J. Ogg, P. B. Kingsley, and J. S. Taylor, Journal of Magnetic RSesonance. Series B 104, 1 (1994), ISSN 10641866 .

[32] F. Träber, W. Block, R. Lamerichs, J. Gieseke, and H. H. Schild, J Magn Reson Imaging 19, 537 (2004), ISSN 10531807.

[33] J. Pauly, P. Le Roux, D. Nishimura, and A. Macovski, IEEE Trans Med Imaging 10, 53 (1991).

[34] H. Barkhuijsen, R. de Beer, and D. van Ormondt, Journal of Magnetic Resonance (1969) 73, 553 (1987), ISSN 00222364 . 
[35] M. H. J. Gruber and M. H. Hayes, Technometrics 39, 335 (1997), ISSN 00401706.

[36] S. W. Provencher, Magnetic Resonance in Medicine 30, 672 (1993), ISSN 0740-3194.

[37] S. Smith, T. Levante, B. Meier, and R. Ernst, Journal of Magnetic Resonance, Series A 106, 75 (1994), ISSN 10641858

[38] S. W. Provencher, LCModel Website, URL http:// s-provencher. com/lcmodel.shtml.

[39] P. B. Roemer, W. A. Edelstein, C. E. Hayes, S. P. Souza, and O. M. Mueller, Magn Reson Med 16, 192 (1990).

[40] M. Guerquin-Kern, L. Lejeune, K. P. Pruessmann, and M. Unser, IEEE Transactions on Medical Imaging 31, 626 (2012), ISSN 02780062, 978-0-87893-286-3.

[41] URL https://www.cibm.ch/cms/lang/fr/pid/60486.

[42] H.-L. M. Cheng and G. A. Wright, Magnetic Resonance in Medicine 55, 566 (2006), ISSN 0740-3194.

[43] S. Liu, R. Fleysher, L. Fleysher, C.-G. Joo, E.-M. Ratai, R. G. González, and O. Gonen, Magnetic Resonance in Medicine 63, 865 (2010), ISSN 07403194.

[44] T. M. Rudkin and D. L. Arnold, Archives of Neurology 56, 919 (1999)

[45] G. Oz, J. R. Alger, P. B. Barker, R. Bartha, A. Bizzi, C. Boesch, P. J. Bolan, K. M. Brindle, C. Cudalbu, A. Dinçer, et al., Radiology (2014), ISSN 1527-1315.

[46] O. Ciccarelli, F. Barkhof, B. Bodini, N. D. Stefano, X. Golay, K. Nicolay, D. Pelletier, P. J. Pouwels, S. A. Smith, C. A. Wheeler-Kingshott, et al., The Lancet Neurology 13, 807 (2014), ISSN 14744465.

[47] M. A. Smith, J. Gillen, M. T. McMahon, P. B. Barker, and X. Golay, Magn Reson Med 54, 691 (2005).

[48] H. Zhu, R. Ouwerkerk, and P. B. Barker, Magn Reson
Med 63, 1486 (2010).

[49] X. Peng, F. Lam, Y. Li, B. Clifford, and Z.-P. Liang, Magnetic Resonance in Medicine 21, 13 (2017), ISSN 07403194

[50] E. Adalsteinsson, P. Irarrazabal, S. Topp, C. Meyer, A. Macovski, and D. M. Spielman, Magnetic Resonance in Medicine 39, 889 (1998), ISSN 07403194.

[51] M. Esmaeili, T. F. Bathen, B. R. Rosen, and O. C. Andronesi, Magnetic Resonance in Medicine 77, 490 (2017), ISSN 15222594.

[52] A. Ebel, B. J. Soher, and A. A. Maudsley, Magnetic Resonance in Medicine 46, 1072 (2001), ISSN 0740-3194.

[53] A. Lecocq, Y. Le Fur, A. A. Maudsley, A. Le Troter, S. Sheriff, M. Sabati, M. Donnadieu, S. Confort-Gouny, P. J. Cozzone, M. Guye, et al., Journal of Magnetic Resonance Imaging 42, 280 (2015), ISSN 10531807.

[54] M. Donadieu, Y. Le Fur, A. Lecocq, A. A. Maudsley, S. Gherib, E. Soulier, S. Confort-Gouny, F. Pariollaud, M.-P. Ranjeva, J. Pelletier, et al., Journal of Magnetic Resonance Imaging 44, 411 (2016), ISSN 10531807.

[55] V. O. Boer, D. W. J. Klomp, C. Juchem, P. R. Luijten, and R. A. de Graaf, Magnetic Resonance in Medicine 68, 662 (2012), ISSN 07403194, NIHMS150003.

[56] B. Strasser, M. Považan, G. Hangel, L. Hingerl, M. Chmelík, S. Gruber, S. Trattnig, and W. Bogner, Magnetic Resonance in Medicine (2017), ISSN 15222594.

[57] L. Hingerl, W. Bogner, P. Moser, M. Považan, G. Hangel, E. Heckova, S. Gruber, S. Trattnig, and B. Strasser, Magnetic Resonance in Medicine 79, 2874 (2018), ISSN 15222594 .

[58] C. Juchem and R. A. de Graaf, Analytical Biochemistry 529, 17 (2017), ISSN 10960309. 


\section{Supporting Informations to Fast high-resolution brain metabolite mapping on a clinical 3T MRI by accelerated ${ }^{1}$ H-FID-MRSI and low-rank constrained reconstruction}

\section{Full Pipeline}

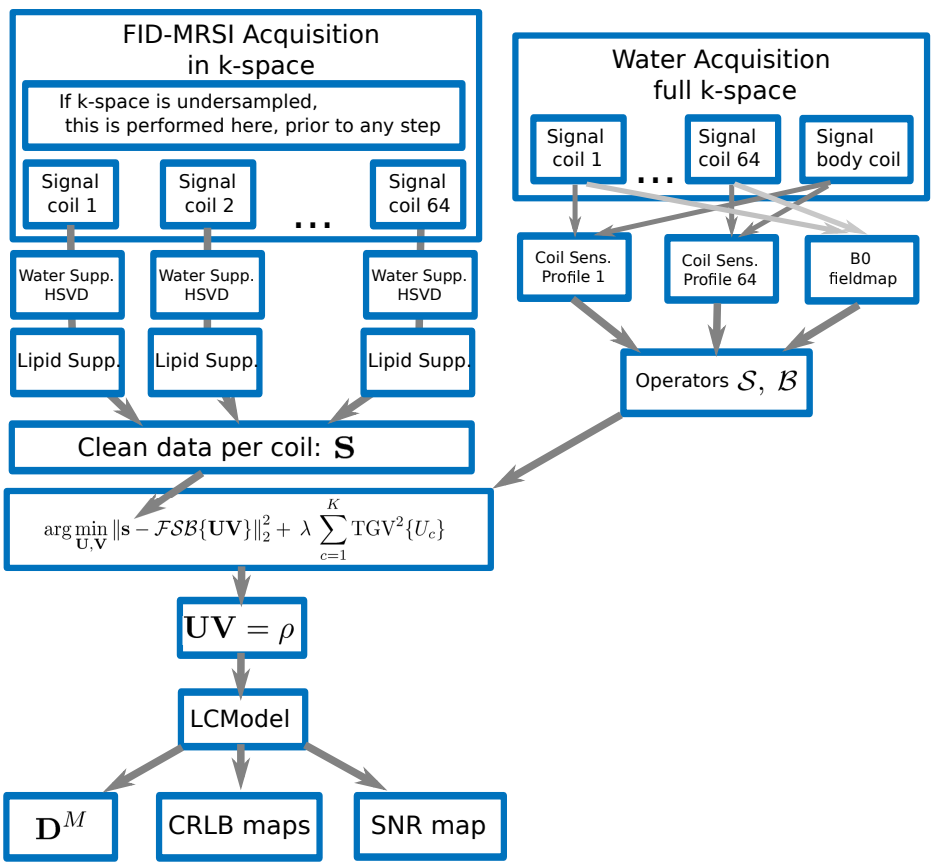

No Model Pipeline

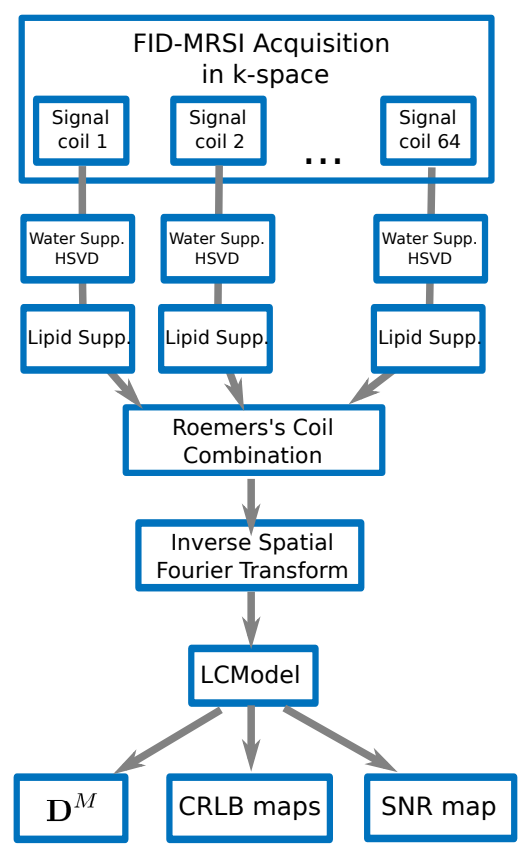

Supporting Information Figure S1.: Sketch of the pipeline used for the reconstruction of acquired MRSI mulit-coil data sets. Pipeline to the left represents the full reconstruction including the low-rank TGV model. To the right, the pipeline without model is presented (this pipeline is used for comparison).
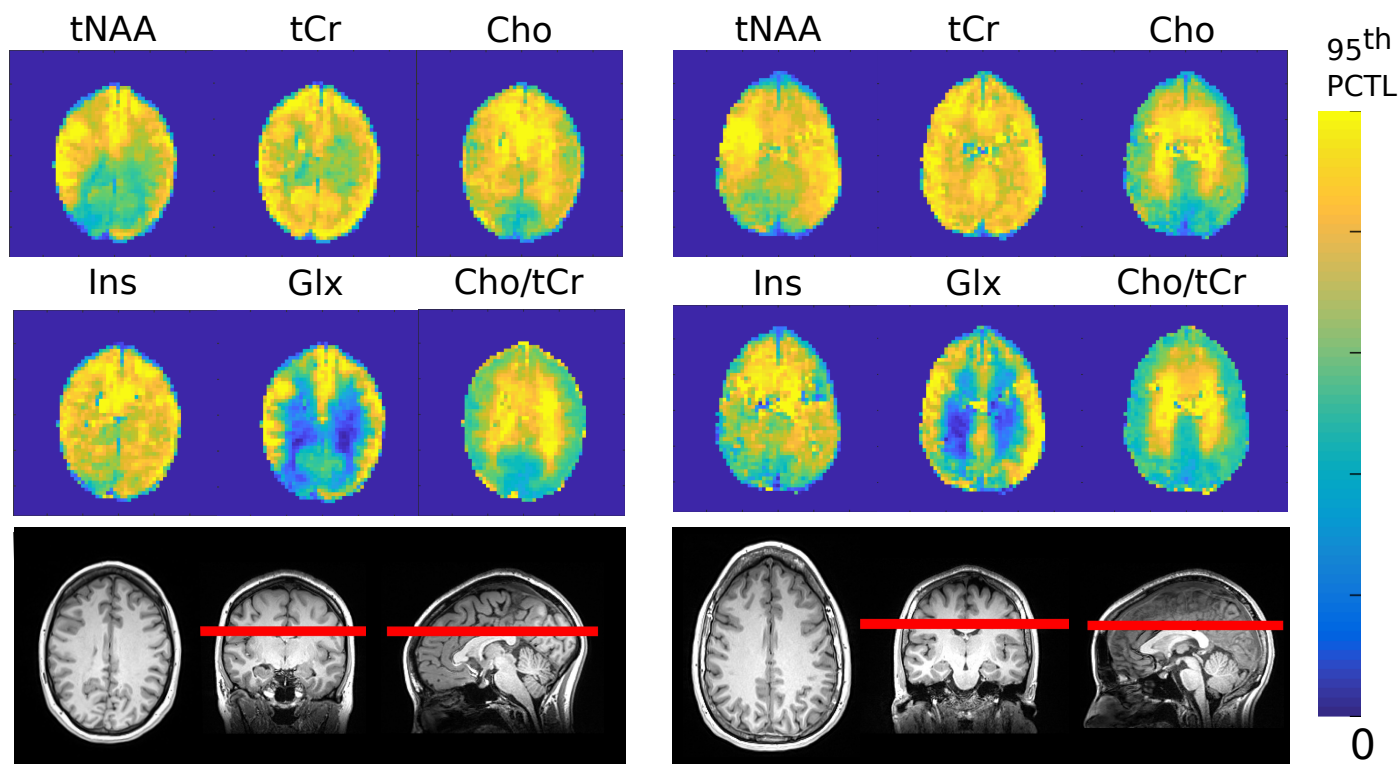

Supporting Information Figure S2.: Metabolite distributions $\boldsymbol{D}^{M}$ from two extra healthy volunteers reconstructed with the low-rank TGV model with the optimal regularization parameter $\left(\lambda=10^{-3}\right)$ and rank $K=20$ without acceleration. The maximum of the scale was determined by the 95th percentile (PCTL) of each metabolite image separately. 


\section{low-rank TGV}

Acc.

Fact.:

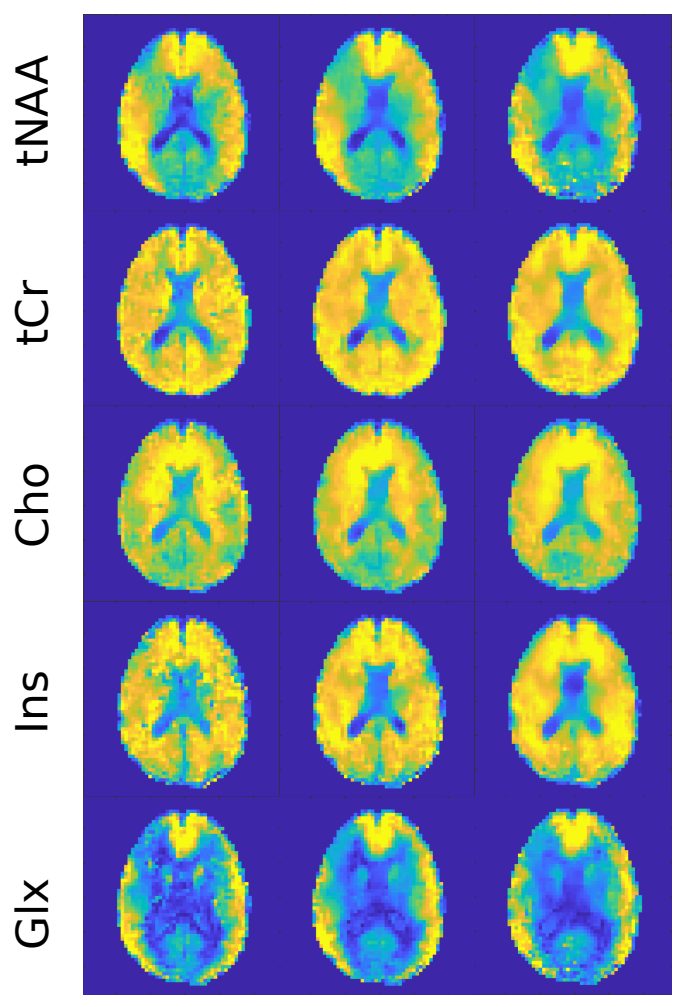

low-rank TV

Acc.

Fact.: 1

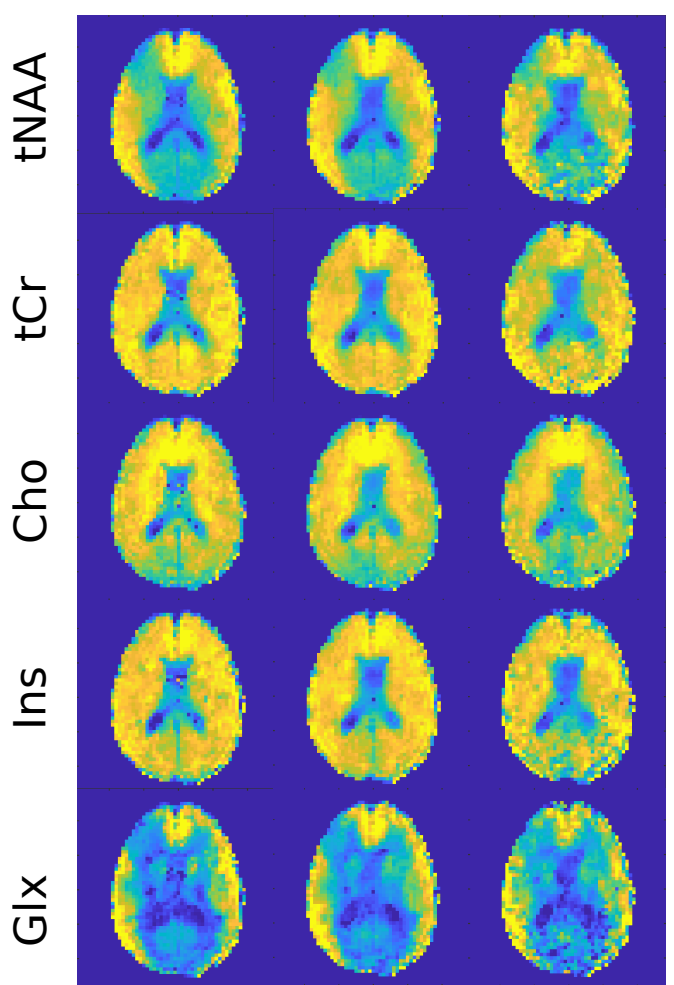

\section{low-rank TGV (static V )}

Acc.

Fact.: 1

2

4

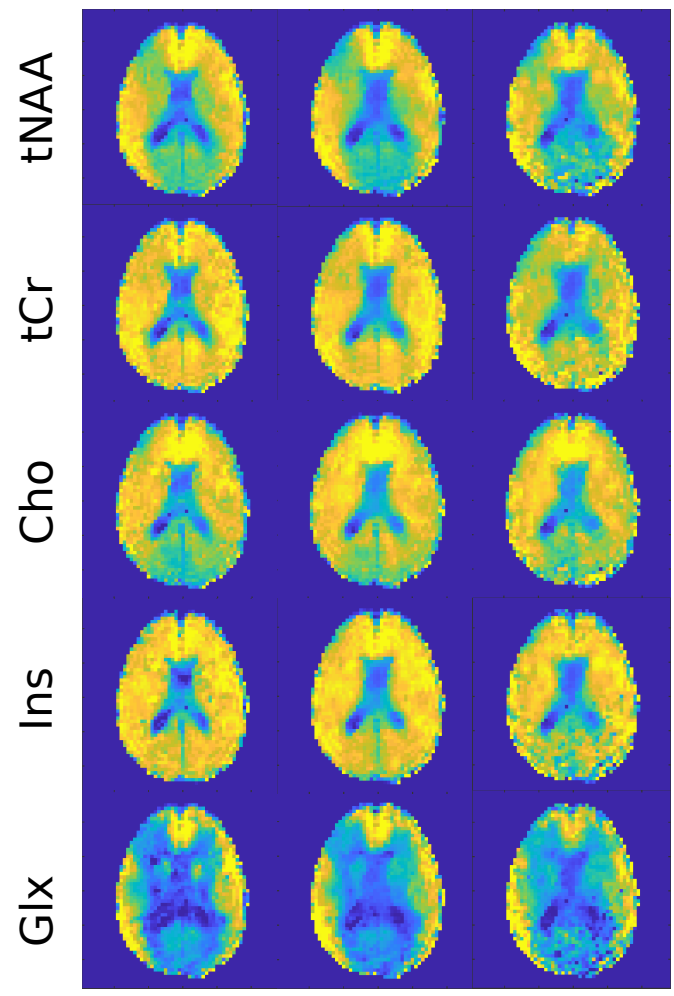

TGV (no low-rank)

Acc.

Fact.: 1

2

4

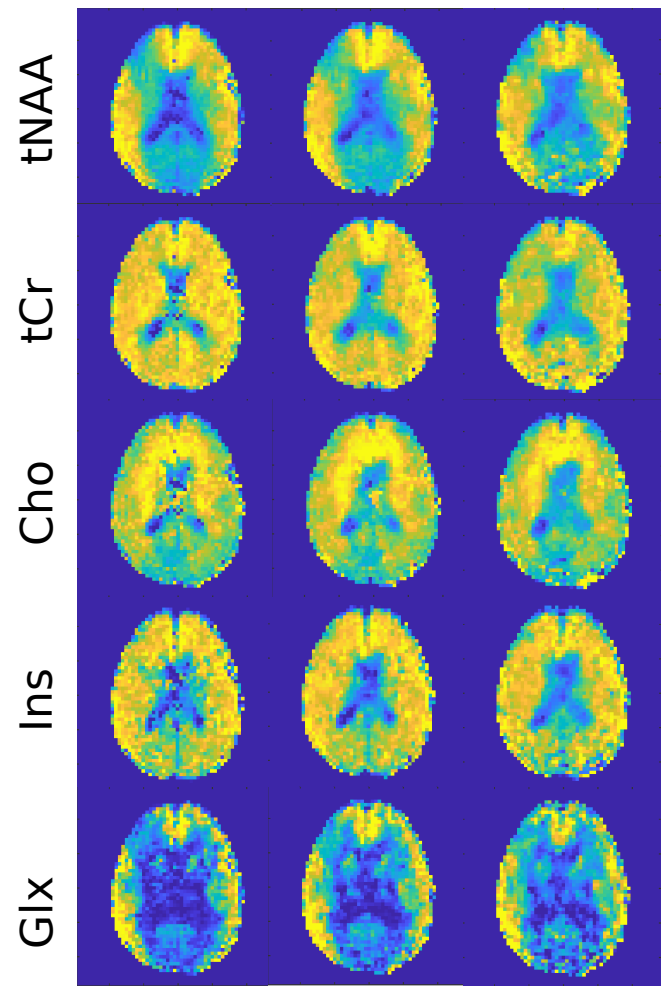

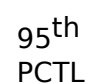

PCTL

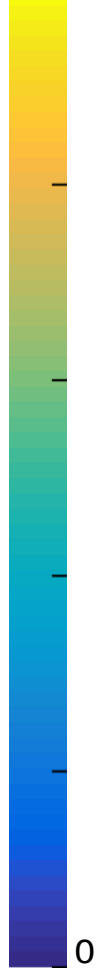

Supporting Information Figure S3.: Metabolite distributions $\boldsymbol{D}^{M}$ reconstructed with different models. low-rank $T G V$ is the model used in this study, low-rank TGV (static $\mathbf{V}$ ) is the same model but with pre-determined time components, low-rank $T V$ is identical but with total variation (TV) regularization and $T G V$ (no low-rank) is a reconstruction model including only TGV regularization without low-rank approximation. Reconstructions were performed with $\lambda=10^{-3}$ and $K=20$ (except no low-rank) and without acceleration or with $\mathrm{AF}=2,4$. 


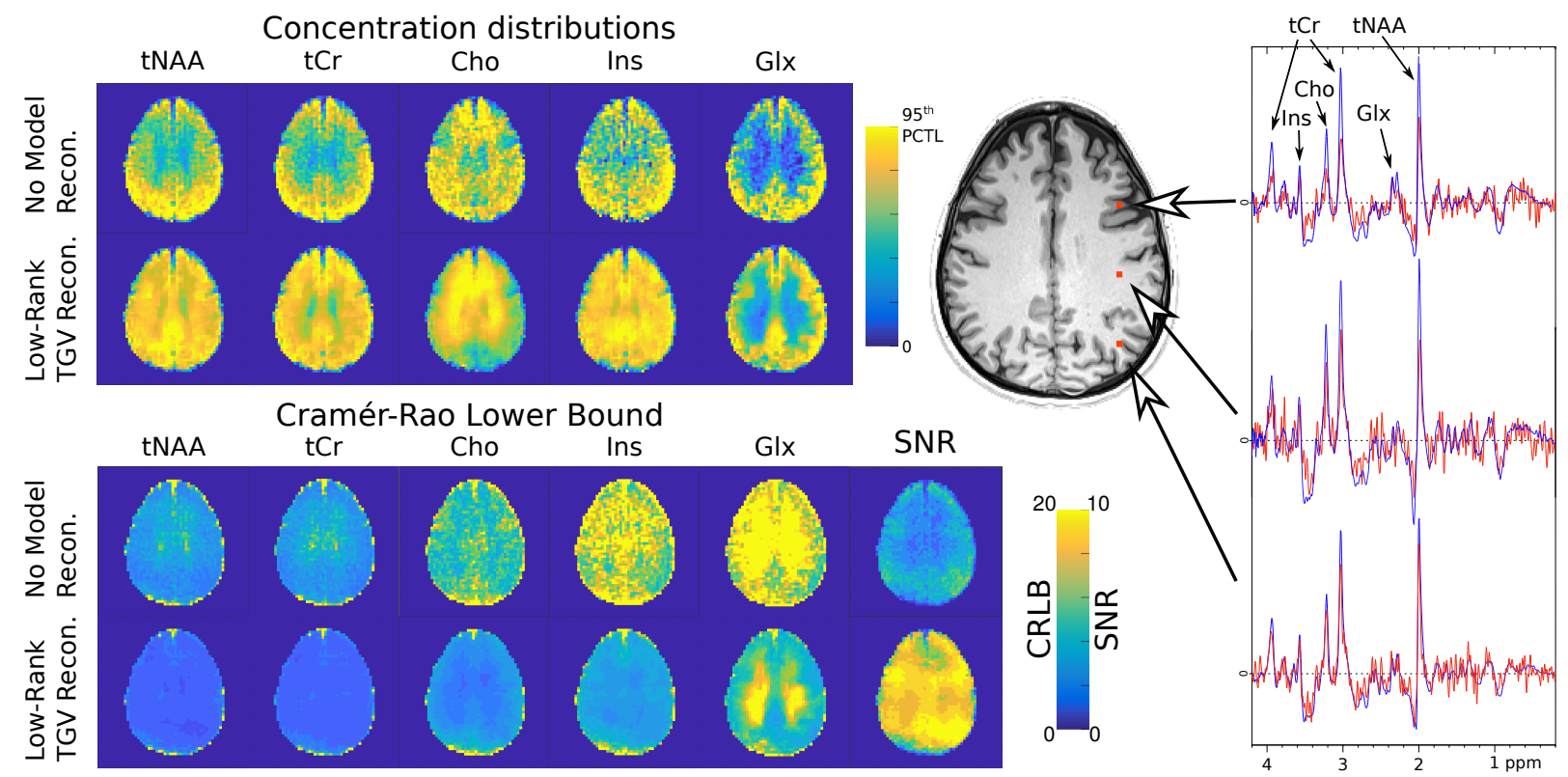

Supporting Information Figure S4. (Extend version of article Fig. 9): Effect of the low-rank TGV reconstruction on the final metabolite distributions $\boldsymbol{D}^{M}$ and spectral quality parameters CRLB and SNR computed from LCModel. For Low-Rank TGV Recon., distributions were reconstructed with the model and $\lambda=10^{-3}, K=20$. The metabolite distributions reconstructed without model (No Model Recon.) result from a pipeline including only lipid suppression and LCModel quantification. The scale ranges from 0 to the 95th percentile (PCTL) of each concentration distribution separately. Three real parts of spectrum samples are shown with their respective locations on the right. The red line belongs to the reconstruction without model and the blue line corresponds to the low-rank TGV reconstruction.

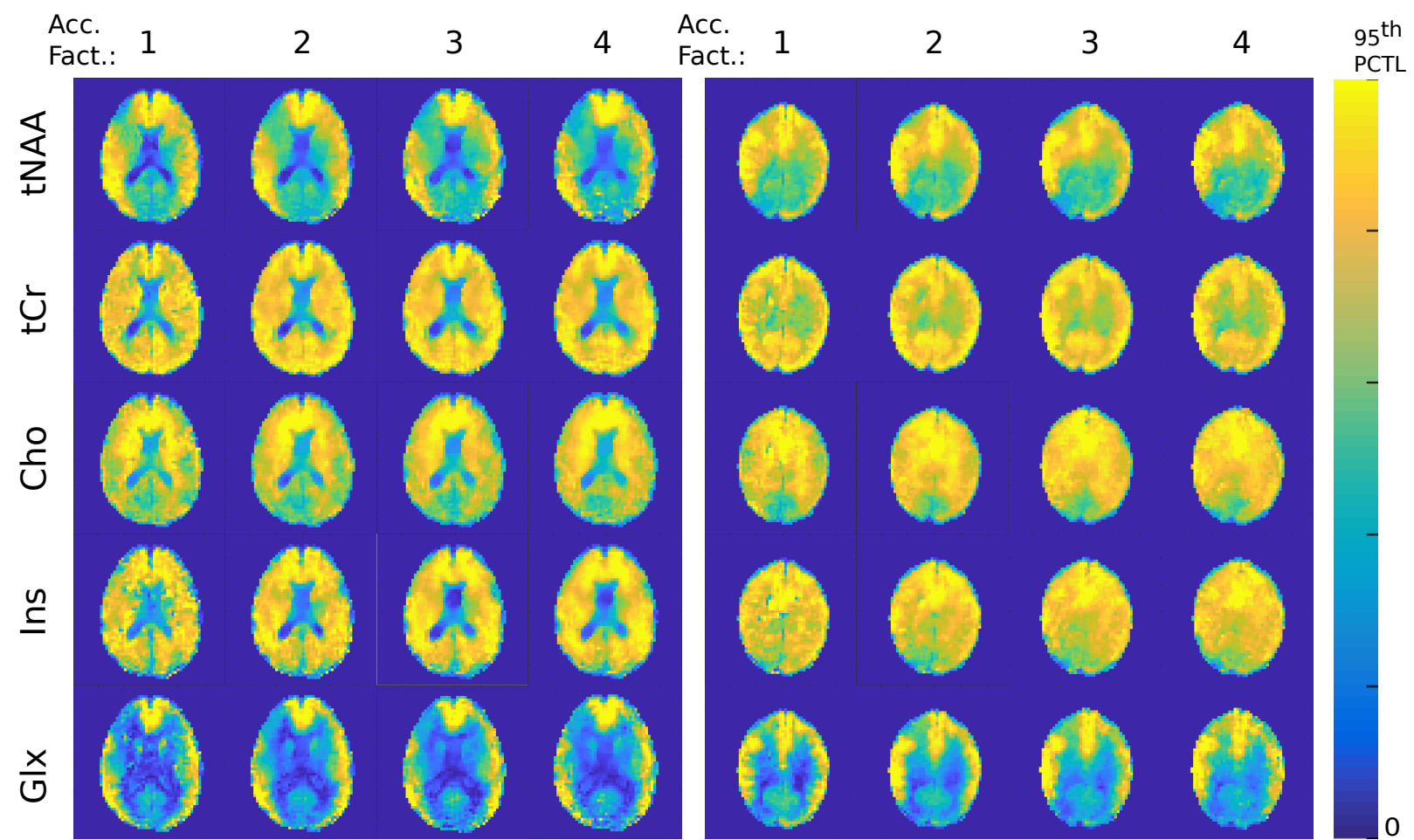

Supporting Information Figure S5. (Extend version of article Fig. 10): Effect of k-space undersampling acceleration on metabolite distribution maps for two in vivo dataset. Both MRSI datasets were reconstructed with rank $K=20$, optimal regularization parameter $\left(\lambda=10^{-3}\right)$ and with acceleration factors $=1,2,3,4$. The scale ranges from 0 to the 95th percentile (PCTL) of each concentration distribution and acceleration factor separately. 

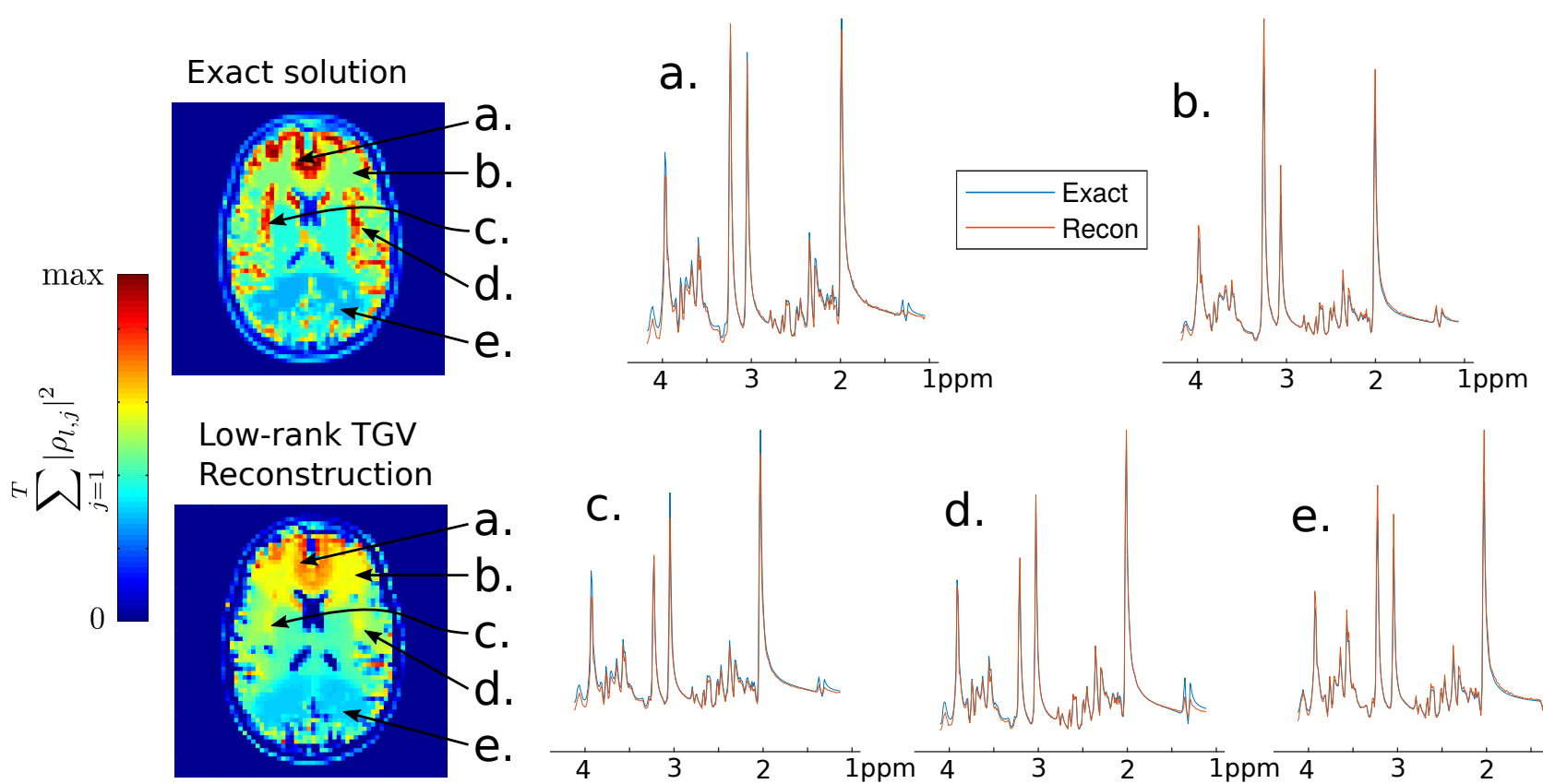

Supporting Information Figure S6.: Sample spectra of the exact solution without noise and the low-rank TGV reconstruction from the simulated MRSI dataset at several locations. Data were reconstructed with $\lambda=5 \cdot 10^{-4}$ and rank

$K=20$ without acceleration. Left, energy image of the analytical exact solution and the low-rank TGV reconstructed solution.

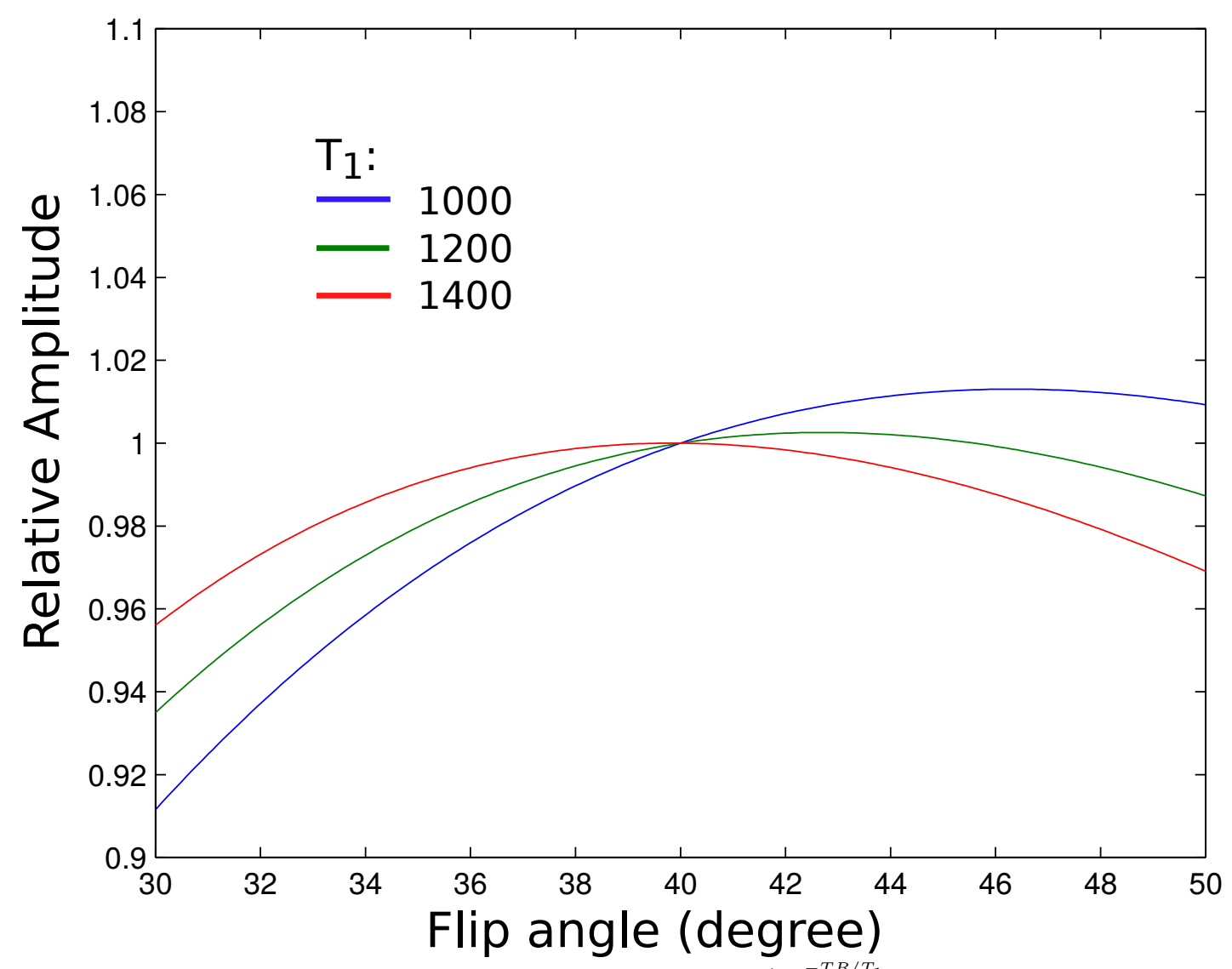

Supporting Information Figure S7.: Steady state signal, $\propto \sin (\alpha) \frac{1-\mathrm{e}^{-T R / T_{1}}}{1-\cos (\alpha) \mathrm{e}^{-T R / T_{1}}}$, computed for flip angle, $\alpha$, ranging from 30 to 50 degree and for $T_{1}=1000,1200$ and $1400 \mathrm{~ms}$. The Ernst angle (40 degree) is located at a saddle point that implies signal to be only slightly dependent on $\alpha$ deviation. 